\title{
Extensional Rheometry of Entangled Solutions
}

\author{
P. K. B hattacharjee, ${ }^{\dagger}$ J . P. Oberhauser, ${ }^{\ddagger, \S}$ G. H. McKinley, ${ }^{\perp}$ L. G. Leal, $, *, \neq, \|$ and \\ T. Sridhar ${ }^{\dagger}$
}

Department of Chemical Engineering, Monash University, Australia;

Department of Chemical Engineering, University of California, Santa Barbara, California;

Department of Mechanical Engineering, Massachusetts Institute of Technology,

Cambridge, Massachusetts; and Materials Department, University of California,

Santa Barbara, California

Received October 25, 2001; Revised Manuscript Received October 1, 2002

\begin{abstract}
The seminal ideas of de Gennes and Doi and Edwards have provided the theoretical framework for much of the recent effort to model the rheological behavior of entangled polymer melts and solutions. Recent theoretical work has incorporated a number of important additions to the basic Doi-Edwards theory, including an explicit description of chain stretch and additional relaxation mechanisms such as contour length fluctuations (CLF) and convective constraint release (CCR). H owever, very little quantitative data has been published on the rheological behavior of entangled systems in strong flows. Hence, a comprehensive examination of the theoretical developments has not been possible. The experiments described in this paper use the filament stretching rheometer to obtain transient extensional stress growth data and steady state uniaxial extensional viscosity data for a number of entangled, narrow molecular weight distribution polystyrene solutions in the strain-rate regime characterized by a significant degree of both chain alignment and stretch. These results are then compared with theoretical predictions for a number of the current generation of reptation-based models, including mechanisms for chain stretching, contour length fluctuations, and convective constraint release. These comparisons demonstrate that when the model parameters are properly obtained from linear viscoelastic measurements, the recent model due to Mead, Larson, and Doi (Macromolecules 1998, 31, 7895) provides quantitative predictions for this class of flows for solutions spanning the complete range from very lightly to highly entangled solutions.
\end{abstract}

\section{Introduction}

Research into the behavior of polymeric fluids has been pursued for over 50 years. The seminal work of Doi and Edwards ${ }^{2-6}$ provided a conceptual framework that has dominated theoretical research on entangled sol utions or melts for the last 20 years. The basic meanfield model of entanglements as a tube of constraints within which a test chain moves is widely accepted in polymer physics and rheology. Indeed, the basic DE model yields a number of important predictions, including: a nonzero second normal stress difference in simple shear flow, reasonable linear viscoel astic features up to moderate frequencies, an approximately correct scaling of the zero shear viscosity and longest relaxation time with the cube of the mol ecular weight, and an accurate description of stress relaxation following a step strain deformation.

Despite these successes, however, the original DE model incorporates a number of idealizations, and these are responsible for some limitations and deficient predictions. Several recent reviews are available. ${ }^{7-9}$ The most obvious limitation is the assumption that chain retraction occurs instantaneously, so that the only effect of chain stretch is the "snap-back" from an affine deformation that is inherent in the universal strain

\footnotetext{
$\dagger$ Monash University.

₹ Department of Chemical Engineering, University of California, Santa Barbara.

$\S$ Present address: Department of Chemical Engineering, University of Virginia, Charlottesville, VA 22904-4741.

$\perp$ Massachusetts I nstitute of Technology.

"Materials Department, University of California, Santa Barbara.
}

tensor, Q. In reality, retraction occurs on time scales up to the Rouse time, $\tau_{R}$, and the lack of an explicit accounting of chain stretch ensures that the original DE model is only applicable to flows in which the strain rate $\dot{\gamma}$ is significantly below $\tau_{\mathrm{R}}{ }^{-1}$.

For the range of strain rates below $\tau_{\mathrm{R}}{ }^{-1}$, the most wellknown failure of the basic DE model is a spurious maximum in the shear stress at a shear rate of $\mathrm{O}\left(\tau_{\mathrm{d}}{ }^{-1}\right)$, where $\tau_{\mathrm{d}}$ is the disengagement time that characterizes the reptation process. This corresponds to the fact that the viscosity in simple shear flow decreases faster than $\dot{\gamma}^{-1}$ in the range $\mathrm{O}\left(\tau_{\mathrm{d}}{ }^{-1}\right) \leq \dot{\gamma} \leq \mathrm{O}\left(\tau_{\mathrm{R}}{ }^{-1}\right)$. Another widely known inaccuracy in the DE model predictions is the fact that the longest relaxation time, as well as the zero shear viscosity, are experimentally observed to increase with the molecular weight to the 3.4-power rather than the predicted rate of $\mathrm{M}^{3}$ (where $\mathrm{M}$ is the polymer molecular weight).

The various limitations and inaccuracies of the basic DE model have led to proposals for a number of important additions to the physics. The need for and benefits from some of these, such as a model for chain stretch in "fast" flows (the addition of chain stretch to the basic DE model produces what is now known as the DEMG model), ${ }^{10-13}$ as well as chain length fluctuations $(C L F)^{14,15}$ and diffusive constraint rel ease, ${ }^{16-18}$ are wellestablished, and the main uncertainties are in the details of the actual models. Others, such as convective constraint re ease $(\mathrm{CCR})^{1,19-23}$ and models that attempt to incorporate tube deformation ${ }^{24-28}$ are less wellestablished, and less is known about their influence on the predictions of the model except that they tend to eliminate or minimize the stress maximum in shear flow. 
Table 1. Solution Characteristics

\begin{tabular}{|c|c|c|c|c|c|c|c|}
\hline solution name & mol wt & $\begin{array}{c}\text { polymer } \\
\text { concn (wt \%) }\end{array}$ & solvent & $\begin{array}{c}\text { zero shear } \\
\text { viscosity }(\mathrm{Pa} \cdot \mathrm{s})\end{array}$ & Z & $\left(\mathrm{Me}_{\mathrm{e}}\right)_{\mathrm{sol}}$ & $\mathrm{G}_{\mathrm{N}}^{0}(\mathrm{~Pa})$ \\
\hline $3.9 \mathrm{M}, 4.9$ wt \% & 3900000 & 4.90 & DOP & 600 & 6.6 & 591000 & 160.4 \\
\hline $3.9 \mathrm{M}, 7.35$ wt $\%$ & 3900000 & 7.35 & DBP & 533 & 14.8 & 263000 & 541 \\
\hline $3.9 \mathrm{M}, 10.0$ wt \% & 3900000 & 10.0 & DEP & 4570 & 27.4 & 142000 & 1360 \\
\hline $3.9 \mathrm{M}, 15.0$ wt \% & 3900000 & 15.0 & DEP & 53000 & 41.1 & 94900 & 3060 \\
\hline $10.2 \mathrm{M}, 6.0$ wt \% & 10200000 & 6.00 & DBP & 9560 & 25.9 & 394000 & 294 \\
\hline
\end{tabular}

One important limitation to continued theoretical development is the relatively small amount of experimental data for narrow molecular weight distribution polymer solutions or melts outside the linear viscoelastic regime. In addition to linear viscoelastic properties, comparisons have been made with DE theory for: step shear strain, ${ }^{29,30}$ shear stress and first normal stress difference during startup of steady shear flow, $31-34$ and rheo-optical measurements for a number of transient shear flows including startup, 35 relaxation, double-step change in shear rate, ${ }^{36}$ and reversing step changes in shear rate $^{37}$ (which have been compared to various versions of the full DEMG/CLF/CCR models in a recent Ph.D. thesis ${ }^{38}$ ). In addition, some rheo-optical data is available for two-dimensional extensional and mixedtype flows in the two and four-roll mill. ${ }^{39}$ A key omission from this list is uniaxial extension, a void that this paper seeks to fill using the filament stretching rheometer devel oped by Sridhar and co-workers..$^{40,41}$ The experimental data that we obtain are compared with predictions from several versions of the reptation-based models.

A unique feature of purely extensional flows is that the mean orientation angle of the polymer chain is fixed; consequently, the stress (or birefringence in the case of rheo-optical experiments) depends only on the degree of segmental orientation and the amount of chain stretch. Hence, comparisons with model predictions are simplified relative to shear and mixed-type flow data where the mean orientation also changes with flow strength. Since the chain stretch mechanism in the reptation-based models has not yet been adequately tested, the uniaxial extensi onal flow data reported here provides a unique opportunity. Not only are we able to achieve strain rates at which the data exhibit demonstrable chain stretching, but the fixed orientation angle inherent in the purely extensional flow also permits an unambiguous test of the basic assumptions underlying the chain stretching portion of the various models. Additionally, we may evaluate the importance of the CLF and CCR modifications for this class of flows.

\section{Experimental Details}

2.1. Solution Preparation. The polymers used in this study are $3.9 \times 10^{6}(3.9 \mathrm{M})$ and $10.2 \times 10^{6}(10.2 \mathrm{M})$ molecular weight polystyrene procured from Polymer Laboratories, Inc. The $3.9 \mathrm{M}$ sample has a polydispersity index $\left(\mathrm{M}_{\mathrm{w}} / \mathrm{M}_{\mathrm{n}}\right)$ of 1.05 , while the $10.2 \mathrm{M}$ sample is slightly more polydisperse $\left(\mathrm{M}_{\mathrm{w}} / \mathrm{M}_{\mathrm{n}}\right.$ $=1.17)$. The polymer was dissolved in diethyl phthalate (DEP), dibutyl phthalate (DBP), or dioctyl phthalate (DOP) solvents. DEP and DBP are reported to be good solvents for polystyrene under ambient conditions $\left(21^{\circ} \mathrm{C}\right)$, while DOP is known to be a $\Theta$ solvent for polystyrene at $22^{\circ} \mathrm{C} .{ }^{42}$

Solution preparation was greatly facilitated by using methylene chloride as a cosolvent. Subsequently, the methylene chloride was readily evaporated over the course of several days. Table 1 lists the compositions of the solutions, which were chosen in order to span the range from lightly entangled (with a minimum of about six entanglements) to highly entangled (about 40 entanglements per chain). In addition, the $3.9 \mathrm{M}$
10.0 wt \% solution and the 10.2 M 6.0 wt \% solution were designed to compare results with fixed number of entangle ments per chain fixed but different polymer molecular weight.

2.2. Experimental Procedure. The experiments were performed with the filament-stretching rheometer developed by Tirtaatmadja and Sridhar ${ }^{40,41}$ in which a sample is held between two rigid disks and subjected to controlled uniaxial extension. This device has been used extensively for measurement of both steady and transient tensile stresses for Boger fluids undergoing uniaxial extensional flow, where the total strain is large enough to ensure that the polymer chains reach a steady-state configuration. ${ }^{43,44}$ Comprehensive reviews of this technique are available, ${ }^{45,46}$ and details of the present system are described by Gupta et al. ${ }^{44}$

All the experiments were conducted at a constant strain rate based on the midpoint diameter of the filament and in a constant temperature room maintained at $21.0 \pm 0.5^{\circ} \mathrm{C}$. The resolution of the transducer and the characteristics of the fluids limited the range of strain (or extension) rates that could be investigated. At low strain rates (bel ow approximately 0.05 $\mathrm{S}^{-1}$ ), the measured force was below the resolution of the transducer. In addition, gravitational effects were significant at low strain rates, causing the fluid to sag. Consequently, some experiments were conducted in a plateau tank, where the filament was immersed in a fluid of comparable density (water in this case) and the filament was stretched horizontally. At strain rates above approximately $5 \tau_{R}$, the filament either ruptured before reaching steady state or the larger tensile forces tended to cause the liquid to peel from the end plate. Between these extrema, the filament was observed to be uniform and generated reproducible steady-state data.

Shear and dynamic (i.e., linear viscoelastic) properties of the solutions were measured in a Rheometrics Fluid spectrometer (RFS II) or a Rheometrics Dynamic analyzer (RDA II) for the more concentrated fluids. Measurements in the RFS II were performed with a $50 \mathrm{~mm}$ titanium cone-and-plate fixture ( $4^{\circ}$ angle), while a $25 \mathrm{~mm}$ steel cone was used in the RDA II. All measurements were taken at $21^{\circ} \mathrm{C}$. The linear viscoelastic (dynamic) data were used to estimate the characteristic relaxation times, as explained in section 4.

Prior to discussing experimental results, however, it is desirable to briefly review the theoretical models mentioned in the Introduction. These will form the basis for comparisons between the experimental data and model predictions and al so guide the determination of relaxation times from the linear viscoelastic data.

\section{Theoretical Background}

As noted in the Introduction, a number of modifications of the original DE theory have been proposed. 1,10-21,24-28 These changes have arisen primarily from recognition of the need for faster relaxation of the flow-induced chain segment orientation distribution as well as the explicit inclusion of a mechanism for chain stretch.

In the present work, we compare measured extensional stress for uniaxial extension with predictions from various extensions of the so-called DEMG model. The latter adds chain stretch to the basic DE model in the form originally suggested by Marrucci and Grizzuti. ${ }^{10,11}$ The various generalizations of the DEMG model that we consider include the addition of CLF (both in the form originally proposed by Doi and 
Kuzuu ${ }^{14,47}$ and in a form adapted from the more recent proposal of Milner and MCLeish ${ }^{15}$ ) and CCR [both the form proposed by Mead, Larson, and Doi (MLD) ${ }^{1}$ and a simplified form adapted from the original proposal of Marrucci and co-workers ${ }^{19-21}$ ]. None of these models includes a mechanism for diffusive constraint release (DCR), which is known to have a major impact on polydisperse systems but has generally been believed to be relatively unimportant for narrow molecular weight distribution polymers like those studied here. However, we shall see a number of discrepancies between our experimental data and model predictions that may reflect contributions from DCR.

A complete summary of the basic DEMG model in the form used here has been published elsewhere. ${ }^{12} \mathrm{Also}$, a number of the generalizations of this model to include CLF and CCR, as well as numerical predictions of their rheol ogical (or rheo-optical) response in specified steady or time-dependent shear flows, can be found in the Ph.D. thesis of Oberhauser.$^{38}$ In the interest of brevity, we will list the governing equations for these various models without much discussion.

3.1. The Basic DEMG Model. The basic DEMG model used here includes the chain stretching mechanism proposed by Marrucci and Grizzutti ${ }^{10,11}$ but with a finitely extensible spring of the form introduced by Mead and Leal ${ }^{12}$ and Mead et al. ${ }^{13}$

The equations describing the relaxation of the chain segment orientation distribution are identical to those originally developed by Doi and Edwards. ${ }^{2-6}$ The key is the convection-diffusion equation for the tube survival probability function, $\mathrm{G}\left(\mathrm{s}, \mathrm{t}, \mathrm{t}^{\prime}\right)$ :

$$
\frac{\partial \mathrm{G}\left(\mathrm{s}, \mathrm{t}, \mathrm{t}^{\prime}\right)}{\partial \mathrm{t}}=\mathrm{D} \frac{\partial^{2} \mathrm{G}}{\partial \mathrm{s}^{2}}-\langle\mathrm{v}(\mathrm{s}, \mathrm{t})\rangle \frac{\partial \mathrm{G}}{\partial \mathrm{s}}
$$

Here, s represents the curvilinear distance al ong the socalled primitive chain measured from the midpoint of the tube, and $\mathrm{D}$ is the one-dimensional diffusivity for reptation:

$$
\mathrm{D}=\mathrm{L}_{0}{ }^{2} / \tau_{\mathrm{d}, 0} \pi^{2}
$$

$L_{0}$ is the equilibrium contour length of the primitive chain, and $\tau_{\mathrm{d}, 0}$ is the characteristic relaxation time scale known as the disengagement time. It is convenient to express $\tau_{\mathrm{d}, 0}$ in terms of the Rouse time for the chain via the relation

$$
\tau_{\mathrm{d}, 0}=3 Z \tau_{\mathrm{R}}=3 Z^{3} \tau_{\mathrm{e}}
$$

where $\mathbf{Z}$ is the average number of entanglements per chain and $\tau_{\mathrm{e}}$ is the Rouse time for a single entanglement segment.

The convection term in eq 1 is calculated using a preaveraged approximation of the velocity of points on the tube relative to the midpoint at $\mathrm{s}=0 .{ }^{2}$

$$
\begin{array}{r}
\langle\mathrm{v}(\mathrm{s}, \mathrm{t})\rangle=\nabla \mathbf{V}(\mathrm{t}): \int_{0}^{\mathrm{s}}\left[\left\langle\mathbf{u}\left(\mathrm{s}^{\prime}, \mathrm{t}\right) \mathbf{u}\left(\mathrm{s}^{\prime}, \mathrm{t}\right)\right\rangle-\frac{1}{3} \mathbf{I}\right] \mathrm{ds^{ \prime }}= \\
\nabla \mathbf{V}(\mathrm{t}): \int_{0}^{\mathrm{s}} \mathbf{s}\left(\mathrm{s}^{\prime}, \mathrm{t}\right) \mathrm{ds^{ \prime }}
\end{array}
$$

The vel ocity gradient tensor for the flow is denoted as $\nabla \mathbf{V}(\mathrm{t})$. The unit vector corresponding to the orientation of a specific primitive chain segment is $\mathbf{u}(\mathrm{s}, \mathrm{t})$. The order parameter tensor $\mathbf{S}(\mathbf{s}, \mathrm{t})$ is the dyadic product uu averaged over the orientation distribution function.
The order parameter tensor $\mathbf{S}(\mathrm{s}, \mathrm{t})$ is calculated pre cisely as described by Doi and Edwards ${ }^{2}$ from $G$ and the so-called universal (strain) tensor $\mathbf{Q}$ :

$$
\mathbf{S}(\mathrm{s}, \mathrm{t})=\int_{-\infty}^{\mathrm{t}} \mathrm{G}\left(\mathrm{s}, \mathrm{t}, \mathrm{t}^{\prime}\right)\left(\frac{\partial \mathbf{Q}\left(\mathbf{E}\left(\mathrm{t}, \mathrm{t}^{\prime}\right)\right)}{\partial \mathrm{t}^{\prime}}\right) d \mathrm{t}^{\prime}
$$

The universal "strain" tensor, $\mathbf{Q}\left(\mathbf{E}\left(t, t^{\prime}\right)\right)$, describes the nonaffine deformation of the freely jointed chain. Although other definitions of the strain tensor have recently been proposed, 48 the models considered here all use the original DE form without the independent alignment approximation. The method by which values of the universal tensor are calculated is described by Mead, Yavich, and Leal (1995). ${ }^{13}$ The boundary conditions on $\mathrm{G}$ are $\mathrm{G}=1$ at $\mathrm{t}=\mathrm{t}^{\prime}$ and $\mathrm{G}=0$ at $\mathrm{s}= \pm \mathrm{L} / 2$.

Chain stretch in the DEMG model is tracked via the one-dimensional tube strain function, $\mathrm{s}\left(\mathrm{s}_{0}, \mathrm{t}\right)$, which maps the deformation of a tube segment from its undeformed reference position so. In the DEMG model, the chain stretch is governed by a simple deterministic equation for the function $\mathrm{s}$ :

$$
\frac{\partial s}{\partial t}=\langle v(s, t)\rangle+3 Z D \frac{\partial^{2} s}{\partial s_{0}^{2}}\left(\frac{1}{3} \frac{{d L^{-1}(x)}_{d x}}{d x}\right)
$$

which represents a balance between frictional stretching, caused by the relative velocity between points on the primitive chain and the tube, and chain retraction model ed in eq 6 via a finitely extensible entropic spring. The latter is approximated using the inverse Langevin function $L^{-1}(x)$, where

$$
L(x) \equiv \operatorname{coth}(x)-\left(\frac{1}{x}\right)
$$

and

$$
x=\sqrt{\frac{Z \partial s\left(s_{0}, t\right)}{N \partial s_{0}}}
$$

In eqs 7 and $8, x$ is the fractional extension of a chain segment, $\mathrm{N}$ is the number of Kuhn steps in a polymer chain, and the ratio $\sqrt{\mathrm{N} / \mathrm{Z}}$ is thus the maximum chain segment extension. The time constant embedded in eq 6 is the Rouse time $\tau_{\mathrm{R}}$. As noted by Doi and Edwards, ${ }^{2}$ the disengagement time $\tau_{\mathrm{d}, 0}$, which appears in eq 1 via the diffusivity $D$, is larger than the Rouse time by a factor $3 Z$. The boundary conditions are $\left(\partial \mathrm{S} / \partial \mathrm{S}_{0}\right)_{\mathrm{s}_{0}= \pm\left(\mathrm{L}_{0} / 2\right)}$ $=1$ and $\left(\partial^{2 \mathrm{~s} /} \partial \mathrm{s}_{0}{ }^{2}\right)_{\mathrm{s}_{0}=0}=0$.

To compare directly with experimental data, we ultimately wish to calculate the stress in terms of the order parameter tensor $\mathbf{S}$ and the tube strain function $\mathrm{s}$. As in previous work, 1,12 the stress tensor is assumed to have the form

$$
\sigma=\frac{15}{4}\left(\frac{\mathrm{G}_{\mathrm{N}}^{0}}{3 \mathrm{~L}_{0}}\right) \int_{-\mathrm{L}_{0} / 2}^{\mathrm{L}_{0} / 2} \mathrm{~L}^{-1}(\mathrm{x}) \sqrt{\frac{\mathrm{N}}{\mathrm{Z}}} \frac{\partial \mathrm{S}\left(\mathrm{s}_{0}, \mathrm{t}\right)}{\partial \mathrm{S}_{0}} \mathbf{S}\left(\mathrm{s}_{0}, \mathrm{t}\right) \mathrm{ds} \mathrm{s}_{0}
$$

where $G_{N}^{0}$ is the plateau modulus. It may be noted that the factor 15/4 appears so that, in the absence of chain stretching (i.e., $\partial \mathrm{S} / \partial \mathrm{S}_{0}=1$ ), the asymptotic value of the shear stress in shear flow is $\sigma_{x y} \sim \mathrm{G}_{\mathrm{N}}^{0}$ for shear rates $\dot{\gamma}$ $\gg \tau_{\mathrm{d}, 0}{ }^{-1}$. The modulus $\mathrm{G}_{\mathrm{e}}$ that appears naturally in the original $D E$ theory is $(15 / 4) \mathrm{G}_{\mathrm{N}}^{0}$ (mistakenly called the 
plateau modulus by Mead and Leal ${ }^{12}$ ). However, this ratio depends specifically on the definition of the strain tensor used in calculating S, as it is 6 rather than 15/4 for the strain tensor of Marrucci et al. ${ }^{48}$

3.2. CCR and CLF Models. (a) Contour Length Fluctuations (CLF). I n addition to reptation, contour length fluctuations (CLF) is an extremely important relaxation mechanism, particularly for lightly or moderately entangled polymeric fluids like those studied here. The early CLF studies of Doi and co-workers ${ }^{14,47}$ suggest that relaxation due to contour length fluctuations becomes exponentially less probable as one moves away from the chain ends. However, Milner and McLeish have recently proposed an alternative scaling, ${ }^{15}$ which improves predictions for the high frequency asymptote of the dynamic loss modulus and gives good agreement with the experimentally observed 3.4-power dependence of the zero shear viscosity and reptation time scale with the polymer molecular weight.

To incorporate the CLF mechanism into the basic DEMG model, we follow the lead of Mead, Larson, and Doi ${ }^{1}$ and add a simple "exponential" relaxation term to the governing equation for the tube survival probability function:

$$
\frac{\partial G\left(s, t, t^{\prime}\right)}{\partial t}=D \frac{\partial^{2} G}{\partial s^{2}}-\langle v(s, t)\rangle \frac{\partial G}{\partial s}-\underbrace{\frac{G}{\tau_{f}\left(s_{0}\right)}}_{\substack{\text { Contour Length } \\ \text { Fluctuations }}}
$$

Two different forms for the CLF time scale function have been utilized in the present work. The first follows the original work of Doi and Kuzuu, ${ }^{14,47}$ assuming an activated relaxation process according to which the CLF relaxation time scales as

$$
\tau_{\mathrm{f}, \mathrm{DK}}\left(\overline{\mathrm{S}}_{0}\right)=\frac{\tau_{\mathrm{R}}}{4} \exp \left[\frac{v Z}{2}\left(1-2 \overline{\mathrm{s}}_{0}\right)^{2}\right]
$$

The variable $\bar{s}_{0}$ is the dimensionless unperturbed tube coordinate $\left(\bar{S}_{0} \equiv \mathrm{S}_{0} / L_{0}\right)$, where the primitive chain end is at $\bar{s}_{0}=1 / 2$ and the chain center is at $\bar{s}_{0}=0$. The constant $v$ that appears in eq 11 is set equal to 1.5. As noted previously by Doi, the CLF mechanism relaxes only a fraction of the chain. The entropic barrier to fluctuations into the tube, which is the basis of eq 11 , produces a rapid increase in $\tau_{\mathrm{f}, \mathrm{DK}}\left(\overline{\mathrm{S}}_{0}\right)$ as we move away from the ends of the chain; hence, $\tau_{\mathrm{f}, \mathrm{DK}}\left(\overline{\mathrm{S}}_{0}\right)$ eventually becomes larger than the relaxation time for reptation. According to Doi, this occurs at a position $\bar{s}_{0}$ equal to

$$
\bar{s}_{\mathrm{d}, \mathrm{DK}}=0.5\left(1-\frac{\mathrm{X}}{\sqrt{\mathrm{Z}}}\right)
$$

where $\mathrm{X}$ is a constant that is known to be larger than 1.47. An important point is that the disengagement time for relaxation by reptation is reduced when the CLF mechanism is active, because the primitive chain can disengage from the tube by moving a distance $L_{0}\left(2 \bar{S}_{d, D K}\right)$ rather than $L_{0}$. Consequently, the effective disengagement time for a model that includes the Doi-Kuzuu version of CLF becomes

$$
\tau_{\mathrm{d}, \mathrm{DK}}=\left(2 \overline{\mathrm{s}}_{\mathrm{d}, \mathrm{DK}}\right)^{2} \tau_{\mathrm{d}, 0}
$$

Here, $\tau_{\mathrm{d}, 0}$ is the original $\mathrm{DE}$ disengagement time that does not account for length scale fluctuations of the primitive chain.

The second theory for CLF that we consider is that due to Milner and MCLeish, ${ }^{15}$ according to which the CLF relaxation process consists of two distinct regimes. At long times, relaxation requires the entropically unfavorable "deep" fluctuations that formed the basis of the earlier theory of Doi (and others). However, at early times, the relaxation process for chain segments near the ends of the tube requires only "shallow" Rouse fluctuations of the tube length, a process that is considerably faster than the activated process associated with deep fluctuations. A formula for the relaxation time of these early modes was derived by Milner and McLeish. ${ }^{15}$ In the present work, this theory is implemented in an approximate form (obtained from an unpublished work of Likhtman), where

$$
\begin{gathered}
\tau_{f, M M}\left(\overline{\mathrm{S}}_{0}\right)=\frac{\tau_{\text {early }}\left(\overline{\mathrm{S}}_{0}\right)}{\exp \left[-3 Z\left(\frac{1}{2}-\overline{\mathrm{S}}_{0}\right)^{2}\right]+\frac{\tau_{\text {early }}\left(\overline{\mathrm{S}}_{0}\right)}{\tau_{\text {late }}\left(\overline{\mathrm{S}}_{0}\right)}} \\
\tau_{\text {early }}\left(\overline{\mathrm{S}}_{0}\right)=\frac{9}{16} \pi^{3}\left[\mathrm{Z}\left(\frac{1}{2}-\overline{\mathrm{S}}_{0}\right)\right]^{4} \tau_{\mathrm{e}}
\end{gathered}
$$

and

$$
\tau_{\text {late }}\left(\overline{\mathrm{s}}_{0}\right)=\tau_{\mathrm{e}} \frac{\pi^{2.5} Z^{1.5}}{8 \sqrt{3}\left(\frac{1}{2}-\overline{\mathrm{s}}_{0}\right)} \exp \left[3 Z\left(\frac{1}{2}-\overline{\mathrm{s}}_{0}\right)^{2}\right]
$$

As one moves from the end of the chain toward the center, the time scale for relaxation via $\operatorname{CLF}, \tau_{\mathrm{f}, \mathrm{Mm}}\left(\overline{\mathrm{S}}_{0}\right)$, increases [rapidly once the entropic barrier to chain retraction is activated and $\left.\tau_{\mathrm{f}, \mathrm{MM}}\left(\overline{\mathrm{S}}_{0}\right) \sim \tau_{\text {late }}\left(\overline{\mathrm{S}}_{0}\right)\right]$, and there is again an effective "cutoff" of the CLF mechanism by reptation. The bare reptation time (without CLF) corresponding to a point $\bar{s}_{0}$ is known from the $D E$ theory to be

$$
\tau_{\mathrm{d}, 0}\left(\overline{\mathrm{s}}_{0}\right)=\sum_{\mathrm{p}, \mathrm{odd}} \frac{4 \tau_{\mathrm{d}, 0}}{\pi \mathrm{p}^{3}} \sin \left(\pi \mathrm{p} \overline{\mathrm{s}}_{0}\right)
$$

Interior segments that have not relaxed by CLF on this time scale $\tau_{\mathrm{d}, 0}\left(\overline{\mathrm{S}}_{0}\right)$ will relax by reptation.

In the Milner-McLeish picture, the transition from CLF to reptation occurs at a value of $\bar{s}_{0}$ that we shall denote as $\overline{\mathrm{S}}_{\mathrm{d}, \mathrm{Mm}}$, and the characteristic time scale for the reptation process is reduced to

$$
\tau_{\mathrm{d}, \mathrm{MM}}=\left(2 \overline{\mathrm{s}}_{\mathrm{d}, \mathrm{MM}}\right)^{2} \tau_{\mathrm{d}, 0}
$$

The point of transition from CLF to reptation is determined by equating $\tau_{\mathrm{d}, 0}\left(\overline{\mathrm{S}}_{0}\right)$, reduced by $\left(2 \overline{\mathrm{S}}_{\mathrm{d}, \mathrm{MM}}\right)^{2}$ according to eq 18 , to the CLF time scale $\tau_{\mathrm{f}, \mathrm{MM}}\left(\overline{\mathrm{S}}_{\mathrm{d}}\right)$ from eq 14 :

$$
\begin{aligned}
\tau_{\mathrm{f}, \mathrm{MM}}\left(\overline{\mathrm{S}}_{\mathrm{d}, \mathrm{MM}}\right)=\tau_{\mathrm{d}, \mathrm{MM}}\left(\overline{\mathrm{S}}_{\mathrm{d}, \mathrm{MM}}\right) \equiv \\
\quad\left(2 \overline{\mathrm{S}}_{\mathrm{d}, \mathrm{MM}}\right)^{2} \sum_{\mathrm{p}, \mathrm{ddd}} \frac{4 \tau_{\mathrm{d}, 0}}{\pi \mathrm{p}^{3}} \sin \left(\pi \mathrm{p} \overline{\mathrm{s}}_{\mathrm{d}, \mathrm{MM}}\right)
\end{aligned}
$$

It can be seen from eq 14 that $\tau_{f, M M}\left(\bar{S}_{0}\right)$ depends strongly on the number of entanglements per chain Z. Indeed, as $Z$ increases, $\tau_{f, M m}\left(\bar{s}_{0}\right)$ increases rapidly, and the CLF mechanism contributes less to the overall relaxation 
process (e.g., the transition point $\bar{s}_{0}=\bar{s}_{d, M M}$ moves closer to the end of the chain).

It should be noted that the implementation of the Milner-McLeish formalism in the form of eqs 10 and 14-16 is an approximation to the exact mathematical solution of the so-called "first passage" problem. In that case, one calculates the time required for Rouse-like motions to achieve for the first time a configuration in which the length of the primitive chain within the tube is any given fraction of its equilibrium length $L_{0}$. A more accurate approximation to the solution of this problem, expressed in terms of the relaxation modulus $\mathrm{G}(\mathrm{t})$ [or equival ently the dynamic moduli $\mathrm{G}^{\prime}(\omega)$ and $\left.\mathrm{G}^{\prime \prime}(\omega)\right]$, has recently been obtained in the linear viscoelastic limit. 49 Although we can calculate $\mathrm{G}(\mathrm{t})$ from the solution of eq 10 by integration over $\mathrm{s}$, it is not clear how to specify $\tau_{f, M M}\left(\bar{S}_{0}\right)$ in eq 10 to replicate this more recent solution and/or achieve a closer approximation to the exact solution of the "first passage" problem. The form we have adopted is known to be accurate for sufficiently large $\mathbf{Z}$ and is actually quite reasonable even for moderate values like those considered here. It is also the most advanced form that has been published until now for application in the nonlinear regime.

(b) Convective Constraint Release (CCR). Diffusive constraint release (DCR) has been postulated as an additional mechanism for relaxation of chain segment orientation in polydisperse polymer solutions or melts for some time. ${ }^{16-18,50-56}$ More recently, Ianniruberto and Marrucci proposed that constraint release can also be driven by the relative "convective" motion between chains in a flowing entangled polymer solution or melt.19-21 This process, which they called convective constraint release (CCR), is envisioned by Marrucci and co-workers as operating in parallel with reptation and other diffusion based relaxation processes. The result is a convective disengagement time, $\tau_{\mathrm{d}, \mathrm{CCR}}(\mathrm{s})$, of the form

$$
\frac{1}{\tau_{\mathrm{d}, \mathrm{CCR}}}=\frac{1}{\tau_{\mathrm{d}, 0}}+\beta\left[\langle\mathrm{v}(\mathrm{s}, \mathrm{t})\rangle-\frac{\partial \mathrm{s}}{\partial \mathrm{t}}\right]_{\mathrm{s}=\mathrm{L} / 2}
$$

which produces an effective diffusion rate in eq 10 by changing the time scale in eq 2 from $\tau_{\mathrm{d}, 0}$ to $\tau_{\mathrm{d}, \mathrm{CCR}}$. It is important to note that this change in the "effective" disengagement time applies only to the diffusive time scale for the tube survival probability function in eq 10. In fact, it is assumed that the chain stretching mechanism is unaffected by this version of CCR, in contrast to the model of Mead et al. ${ }^{1}$ that will be discussed momentarily.

The parameter $\beta$ that appears in eq 20 is an empirical numerical coefficient. Ianniruberto and Marrucci showed that $\beta \geq 2.76$ eliminates the spurious shear stress maximum that exists in the DE model without any CCR contribution. Our implementation of eq 20 differs in one important way from the original model of I anniruberto and Marrucci in that the Rouse time $\tau_{\mathrm{R}}$ is set as a lower bound for $\tau_{\mathrm{d}, \mathrm{CCR}}(\mathrm{s})$, such that

$\left.\tau_{\mathrm{d}, \mathrm{CCR}}=\max \left\{\tau_{\mathrm{R}}, \tau_{\mathrm{d}, \mathrm{O}} \mathrm{d} \mid 1+\tau_{\mathrm{d}, 0} \beta\left[\langle\mathrm{v}(\mathrm{s}, \mathrm{t})\rangle-\frac{\partial \mathrm{S}}{\partial \mathrm{t}}\right]_{\mathrm{s}=\mathrm{L} / 2}\right)\right\}$

Equation 21 simply imposes the physically obvious constraint that the Rouse time scale for an unentangled chain ultimately limits the chain relaxation time.

3.3. The Mead-Larson-Doi (MLD) Model. Finally, an alternative and more comprehensive imple- mentation of the principles of convective constraint release and contour length fluctuations, recently published by Mead, Larson, and Doi, ${ }^{1}$ incorporates the CCR mechanism directly into the basic DE MG formalism. In this model (which we will refer to as MLD), eqs 1 and 6 become

$$
\begin{aligned}
& \frac{\partial G\left(s, t, t^{\prime}\right)}{\partial t}=D \frac{\partial^{2} G}{\partial s^{2}}-\langle v(s, t)\rangle \frac{\partial G}{\partial s}+ \\
& \underbrace{g\left(\frac{\partial s}{\partial s_{0}}\right)\left[\frac{2}{L(t)}(\dot{s}-\langle v(s, t)\rangle)_{s=\frac{L}{2}}\right]}_{\text {Convective Constraint Release }}-\underbrace{\frac{G}{\tau_{\xi}\left(s_{0}\right)}}_{\begin{array}{c}
\text { Contour Length } \\
\text { Fluctuations }
\end{array}}
\end{aligned}
$$

and

$$
\begin{aligned}
\frac{\partial s}{\partial t}=\langle v(s, t)\rangle+3 Z D \frac{\partial^{2} s}{\partial s_{0}^{2}}\left(\frac{1}{3} \frac{d \mathrm{~L}^{-1}(x)}{d x}\right)- \\
\underbrace{\frac{1}{2}\left[\frac{2}{L(t)}(\langle v(s, t)\rangle-\dot{s})_{s=\frac{L}{2}}\right]\left(s-s_{0}\right)}_{\text {Convective Constraint Release }},
\end{aligned}
$$

where $D$ is again the original diffusivity given by eq 2 .

The detailed origin of this model is discussed in the original MLD paper. ${ }^{1}$ Here, we recount a few basic points in order to better understand the model comparisons that will be presented later in this paper. First, in contrast to the simplified approach of Ianniruberto and Marrucci, ${ }^{19}$ elements of a convective constraint release mechanism are present in both the tube strain function and tube survival probability function equations. In addition to enhancing the rate of relaxation of chain orientation, Mead et al. argue that the loss of entanglements also facilitates the relaxation of chain stretch. In fact, the transition from CCR relaxation of orientation to stretch is controlled via a so-called "switch function" $g\left(\partial s / \partial s_{0}\right)$. Mead et al. present two versions of the switch function, one that is "self-consistent"

$$
g\left(\partial s / \partial s_{0}\right)=1 /\left(\partial s / \partial s_{0}\right)
$$

and a second that is "ad hoc"

$$
g\left(\partial s / \partial s_{0}\right)=\exp \left[-\left(\partial s / \partial s_{0}-1\right)\right]
$$

All computations presented in this paper, unless otherwise noted, are performed with the self-consistent form of the switch function, which is more appropriate at high strain rates and somewhat more accurate when compared with the experimental data. When chain stretching is significant, $\partial \mathrm{s} / \partial \mathrm{s}_{0} \gg 1$ and $\mathrm{g}\left(\partial \mathrm{s} / \partial \mathrm{s}_{0}\right) \rightarrow 0$ so that CCR preferentially relaxes chain stretch and has virtually no effect on the tube survival probability function; conversely, if the chain is unstretched, then $\partial \mathrm{s} / \partial \mathrm{s}_{0} \approx 1$ and $\mathrm{g}\left(\partial \mathrm{s} / \partial \mathrm{s}_{0}\right) \rightarrow 1$, and the decay of the tube survival probability function is enhanced by CCR.

It should al so be noted that the version of CCR given by eqs 22 and 23 is linear in S and $G$ and thus contributes equally to constraint release over the entire length of the chain. The Marrucci approach, on the other hand, simply replaces the reptation time scale with an effective reptation time given by eq 21 . In this case, the 
strongest effect of CCR is felt at the ends of the chain, similar to the basic reptation process. Although the MLD implementation is ostensibly more comprehensive, both the Marrucci and MLD approaches are essentially ad hoc, and we compare both with the experimental data obtained in this study. A more fundamental, "molecular" approach to modeling CCR has recently been proposed by Milner et al., ${ }^{23}$ but it has not yet been extended to the chain stretching flow regime that is a primary focus of the present study.

Last, none of the models discussed here incorporate diffusi ve constraint release(DCR). This mechanism has generally been assumed to play an important role only for polydisperse systems. H owever, very recent work by Likhtman and McLeish ${ }^{49}$ has shown that certain features of the linear viscoelastic spectrum are qualitatively changed by DCR even for a monodispersepolymer. Nevertheless, our purpose is a quantitative comparison with MLD and related models that do not contain DCR. In any case, we believe that our general conclusions concerning chain stretch and other "strong flow" features will remain valid whether or not the model employed contains DCR, a point to which we will return when we compare model predictions with extensional flow data.

\section{Parameter Estimation}

A critical issue in the comparison of the experimental data for extensional stresses with predictions from the various versions of the reptation model is the accurate determination of model parameters. The parameters relevant to the various theories are the plateau modulus $\mathrm{G}_{\mathrm{N}}^{0}$, the relaxation times $\tau_{\mathrm{R}}$ and $\tau_{\mathrm{d}, 0}$, and the average number of entanglements per chain $Z$. Of these parameters, three are independent since the number of entangl ements $Z$ determines the ratio of the relaxation times $\tau_{\mathrm{R}}$ and $\tau_{\mathrm{d}, 0}$. All of the above are functions of the concentration and molecular weight of the polymer in solution. In the present work, we estimate these parameters via a combination of established literature correlations and linear viscoelastic data.

4.1 Estimation of $\mathbf{Z}$ and $\mathbf{G}_{\mathrm{N}}^{\mathbf{0}}$. In polymer melts, the constraining tube (or the primitive chain) is described by a contour length $\mathrm{L}$ and a step length (or tube diameter) a, while the polymer chain is characterized by the number of Kuhn steps (or statistical segments) $\mathrm{N}$ of length $\mathrm{b}$. The requirement that the mean square end-to-end length of the polymer chain within the tube be identical to the contour length of the tube leads to the relationship $\mathrm{L}=\mathrm{Nb}^{2} / \mathrm{a}$. The average number of entanglements per chain is then $\mathrm{Z}=\mathrm{L} / \mathrm{a}=\mathrm{Nb}^{2} / \mathrm{a}^{2}$. Finally, the length scale $a$ is related to the plateau modulus ${ }^{2}$ as $a^{2}=4 \rho \mathrm{RTNb}^{2} / 5 \mathrm{MG}_{N}^{0}$, where $\rho$ is the polymer melt density, $\mathrm{R}$ is the universal gas constant, $\mathrm{T}$ is the absolute temperature, and $\mathrm{M}$ is the polymer molecular weight. By eliminating $a^{2}$ from the definition of $Z$, we can relate $Z$ and $G_{N}^{0}$ by the relationship $Z=$ $\mathrm{M} /\left(4 \rho \mathrm{RT} / 5 \mathrm{G}_{\mathrm{N}}^{0}\right)$. The denominator is usually referred to as $\mathrm{M}_{\mathrm{e}}$, the molecular weight between entanglements. 57,58 For polystyrene melts, the plateau modulus is $2 \times 10^{5}$ $\mathrm{Pa}$, and the value of $\mathrm{M}_{e}$, defined as

$$
\mathrm{M}_{\mathrm{e}} \equiv 4 \rho \mathrm{RT} / 5 \mathrm{G}_{\mathrm{N}}^{0}
$$

is 13 300. An alternate, commonly employed definition for $\mathrm{M}_{\mathrm{e}}$ (where $\mathrm{M}_{\mathrm{e}} \equiv \rho \mathrm{RT} / \mathrm{G}_{\mathrm{N}}^{0}$ ) has also been suggested by Ferry. ${ }^{59}$ In our analysis, we use the definition of Fetters et al., ${ }^{57}$ which is consistent with the ratio $\tau_{\mathrm{d}, 0} /$ $\tau_{\mathrm{R}}=3 Z$, where

$$
Z=M / M_{e}
$$

The existence of two definitions for $M_{e}$ (and hence $Z$ ) has led to much confusion in the literature.

We now examine the scaling of $\mathrm{M}_{\mathrm{e}}$ with concentration for polymer solutions. For sufficiently large polymer concentrations, the effect of solvent quality becomes negligible, and the scaling of the molecular weight between entanglements with concentration is well established as ${ }^{34}$

$$
\left(\mathrm{M}_{\mathrm{e}}\right)_{\mathrm{sol}} \sim(\mathrm{c} / \rho)^{-1}
$$

where $\mathrm{c}$ is the polymer concentration in solution measured in the same units as $\rho$. For polystyrene, the crossover to this concentrated regime occurs for c greater than $0.1 \mathrm{~g} / \mathrm{cm}^{3}$. Thus, for the concentrated regime (c > $0.1 \mathrm{~g} / \mathrm{cm}^{3}$ ), the polymer exhibits Rouse scaling without hydrodynamic interaction:

$$
\left(\mathrm{M}_{\mathrm{e}}\right)_{\mathrm{sol}}=13300(\mathrm{c} / \rho)^{-1}
$$

which is consistent with the estimate of $\mathrm{M}_{\mathrm{e}}=13300 \mathrm{in}$ the melt.

On the other hand, for highly entangled polymers in the "semidilute" range (concentrations bel ow approximately $0.1 \mathrm{~g} / \mathrm{cm}^{3}$ for polystyrene), the nature of the solvent plays a more important role as evidenced by the dependence of the scaling law between $\mathrm{M}_{\mathrm{e}}$ and $\mathrm{c}$ on the excluded volume parameter $v$. According to the "blob" scaling hypothesis, ${ }^{60}$ the molecular weight between entanglements for polymer solutions may be written as

$$
\left(\mathrm{M}_{\mathrm{e}}\right)_{\mathrm{sol}}=\mathrm{K}(\mathrm{c} / \rho)^{1 /(1-3 v)}
$$

where $\mathrm{K}$ is a constant dependent upon the polymer concentration. If we assume that the solvent is a $\Theta$ solvent, then $v=1 / 2$, and the constant $\mathrm{K}$ for this regime can be estimated by requiring the blob estimate of $\mathrm{Me}_{\mathrm{e}}$ with $v=1 / 2$ to predict the same $\left(\mathrm{M}_{\mathrm{e}}\right)_{\mathrm{sol}}$ at $\mathrm{c}=0.1 \mathrm{~g} / \mathrm{cm}^{3}$ as eq 29. Hence, for the special case of polystyrene in a $\Theta$ solvent (where $c \leq 0.1 \mathrm{~g} / \mathrm{cm}^{3}$ ), we obtain

$$
\left(\mathrm{M}_{\mathrm{e}}\right)_{\mathrm{sol}}=1240(\mathrm{~d} \rho)^{-2}
$$

In the present work, we estimate $\left(\mathrm{M}_{\mathrm{e}}\right)_{\text {sol }}$ for different concentrations using either eq 29 or 31 . The parameters $\mathrm{G}_{\mathrm{N}}^{0}$ and $\mathrm{Z}$ are then calculated from eqs 26 and 27 with $\left(\mathrm{M}_{\mathrm{e}}\right)_{\text {sol }}$ replacing $\mathrm{M}_{\mathrm{e}}$. The resulting estimates of $Z$, $\left(\mathrm{M}_{\mathrm{e}}\right)_{\text {sol }}$, and $G_{N}^{0}$ for each polymer solution are listed in Table 1 . It is evident from the development above that solvent quality plays an important role in the estimation of $\left(\mathrm{M}_{\mathrm{e}}\right)_{\mathrm{sol}}$, and hence $\mathrm{Z}$, for solutions in the semidilute regime. Of the solvents used in this work, DOP is known to be a $\Theta$ solvent for polystyrene at room temperature. The solvent quality of DEP and DBP is perhaps questionable. However, this uncertainty affects $Z$ only for solutions with $c \leq 0.1 \mathrm{~g} / \mathrm{cm}^{3}$ (i.e., the $3.9 \mathrm{M}, 7.35$ wt \% and $10.2 \mathrm{M}, 6.0$ wt \% solutions).

It should be noted that the values listed for $G_{N}^{0}$ in Table 1 are qualitatively consistent with estimates that can be made from linear viscoelastic data for these solutions via ad hoc procedures. The available linear 


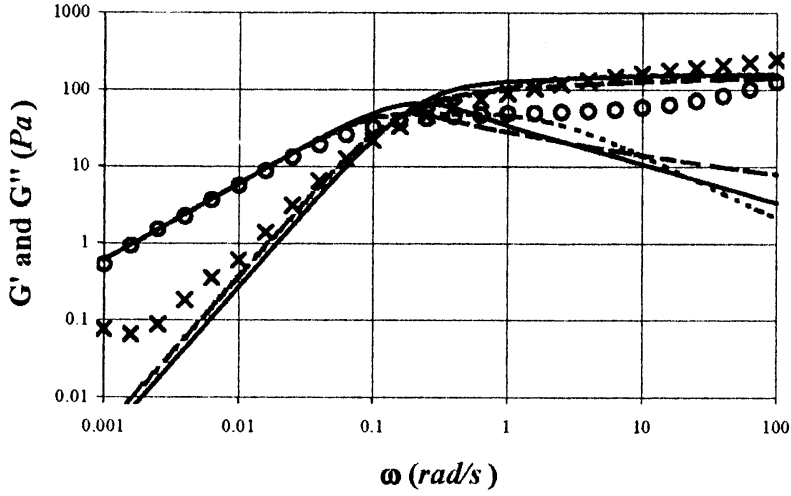

Figure 1. Linear viscoelastic data for the $3.9 \mathrm{M} 4.9$ wt \% solution, where the fits are for the DE $(-), D E / M i l n e r-$ McLeish CLF (- -), and DE/Doi-Kuzuu CLF (-- -) models.

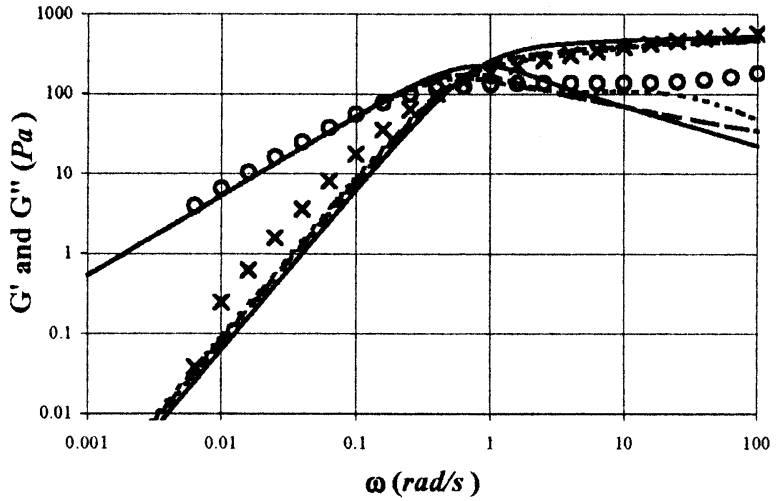

Figure 2. Linear viscoelastic data for the $3.9 \mathrm{M} 7.35$ wt \% solution, where the fits are for the DE $(-)$, DE/MilnerMcLeish CLF $(--)$, and DE/Doi-Kuzuu CLF (-- ) models.

viscoelastic data are shown in Figures $1-5$. Also shown in these plots are various theoretical predictions that we will discuss in the next section. Here, we seek only to contrast our estimates of $G_{N}^{0}$ with values obtained from ad hoc procedures. F or example, one can estimate $\mathrm{G}_{\mathrm{N}}^{0}$ either from the local minimum value of $\mathrm{G}^{\prime \prime}$, which is approximately $1 / 4$ of $\mathrm{G}_{\mathrm{N}}^{0},{ }^{61,62}$ or by implementing a qualitative version of the procedure followed by Osaki and co-workers. ${ }^{61}$ The latter method involves equating $\mathrm{G}_{\mathrm{N}}^{0}$ with the value of $\mathrm{G}^{\prime}$ at the frequency corresponding to the initial (high frequency) upturn in $\mathrm{G}^{\prime \prime}$. To the extent that the available data is sufficient to allow these procedures to be implemented, the resulting estimates of $\mathrm{G}_{\mathrm{N}}^{0}$ are within $10 \%$ of the values given in Table 1 . However, in view of the limited range of the available linear viscoelastic data (F igures $1-5)$, we view these ad hoc estimates as less reliable than those obtained by the well-established literature correlations presented above.

4.2. Estimates of Relaxation Times. The other critical parameter necessary for meaningful comparisons between the experimental data and model predictions is a relaxation time. In the present work, we estimate relaxation times di rectly from the linear viscoelastic data shown in Figures 1-5. As noted earlier, it is sufficient to determine either $\tau_{\mathrm{R}}$ or $\tau_{\mathrm{d}, 0}$ since they are related via the relationship $\tau_{\mathrm{d}, 0}=3 Z \tau_{\mathrm{R}}$. The longest Rouse time for the chain, $\tau_{\mathrm{R}}$, is related to the Rouse time for a single segment between entanglement points [i.e., the classical Rouse time, $\tau_{\mathrm{e}}$, for a chain with molecular weight $\left(\mathrm{M}_{\mathrm{e}}\right)_{\text {sol }}$ in the same solvent] via the relationship $\tau_{\mathrm{R}}=\mathrm{Z}^{2} \tau_{\mathrm{e}}$.
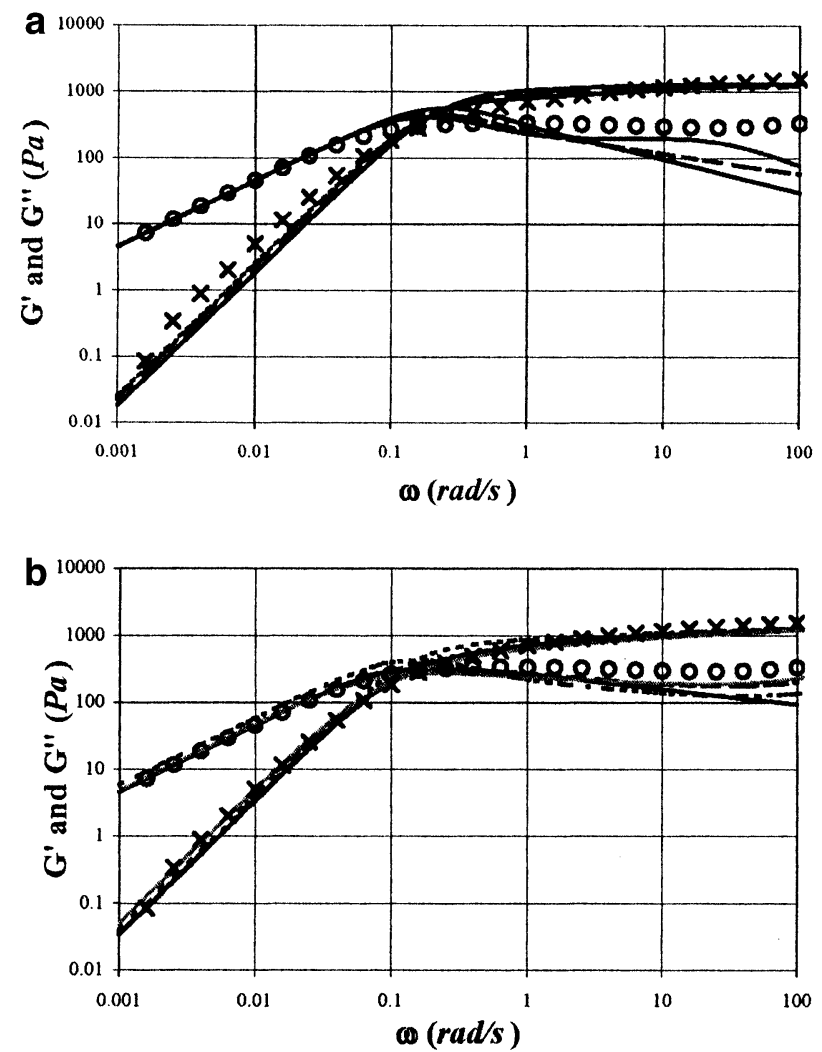

Figure 3. (a) Linear viscoelastic data for the $3.9 \mathrm{M} 10.0 \mathrm{wt}$ $\%$ solution, where the fits are for the DE (-), DE/MilnerMCLeish CLF $(--)$, and DE/Doi-Kuzuu CLF (- - ) models. (b) Linear viscoelastic data for the $3.9 \mathrm{M} 10.0$ wt \% solution, where the fits are for the LV Milner-McLeish CLF model without Rouse modes $(-)$, the LV Milner-McLeish CLF model with Rouse modes $(--)$, the LV Likhtman-McLeish CLF model without DCR (-- ), and the LV Likhtman-McLeish CLF model with DCR (gray line).

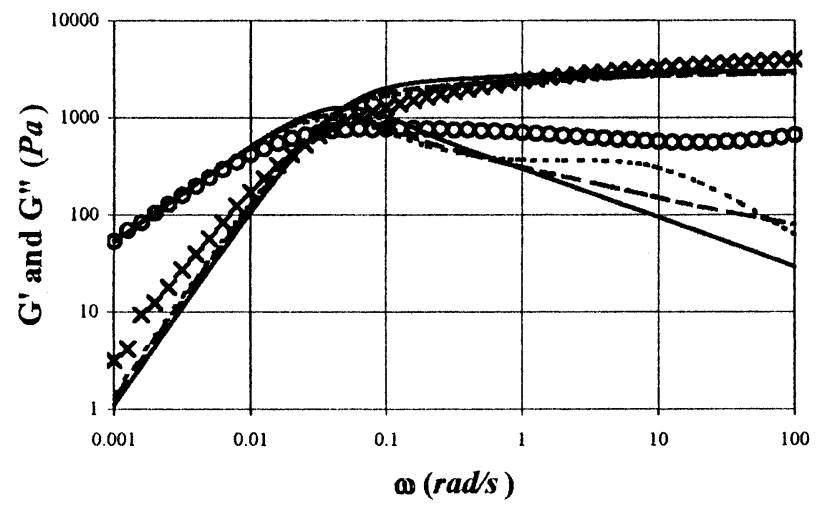

Figure 4. Linear viscoelastic data for the $3.9 \mathrm{M} 15.0 \mathrm{wt} \%$ solution, where the fits are for the DE $(-)$, DE/MilnerMcLeish CLF (- -$)$, and DE/Doi-Kuzuu CLF (-- ) models.

In estimating relaxation times, it is important to remember that the longest measurable relaxation time (often called the terminal time and denoted $\tau_{\text {term }}$ ) is reduced significantly from the "true" reptation time, $\tau_{\mathrm{d}, 0}$, by contributions from other relaxation mechanisms such as chain length fluctuations and diffusive constraint rel ease that are active at low frequency [cf. eq 13 or 18]. Since reliable data often does not exist at sufficiently high frequencies to obtain a direct estimate of the Rouse time, estimates of relaxation times are commonly based on the low-frequency linear viscoelastic data. However, the best estimates of the time scales $\tau_{\mathrm{R}}$ and $\tau_{\mathrm{d}, 0}$ are then 


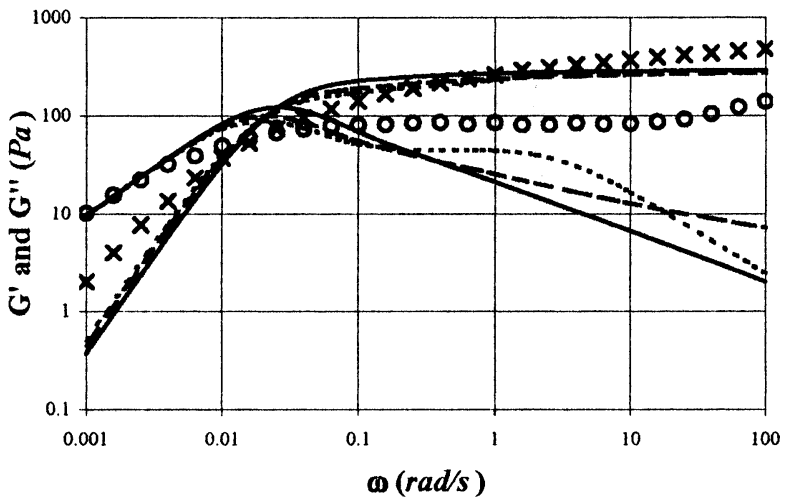

Figure 5. Linear viscoelastic data for the $10.2 \mathrm{M} 6.0 \mathrm{wt} \%$ solution, where the fits are for the DE $(-), D E / M i l n e r-$ McLeish CLF (- -), and DE/Doi-Kuzuu CLF (-- -) models.

model dependent, resulting in a great deal of uncertainty about the most reliable procedures for inferring time scales. One factor is that the predicted relationship between $\tau_{\text {term }}$ and $\tau_{\mathrm{d}, 0}$ depends on the model. Indeed, in a recent paper, Osaki showed that apparently equally reliable parameter estimates for $\tau_{\mathrm{R}}$ from a single set of data can differ by at least a factor of $5 !^{63}$

In the simple DE or DEMG models, $\tau_{\mathrm{d}, 0}$ is equal to $\tau_{\text {term. }}$. However, if we consider a model that contains CLF (but not DCR), the longest measurable relaxation time, $\tau_{\text {term, }}$, is denoted as either $\tau_{\mathrm{d}, \mathrm{DK}}$ or $\tau_{\mathrm{d}, \mathrm{Mm}}$ [if one uses either eq 13 or 18, respectively] and may be significantly smaller than $\tau_{\mathrm{d}, 0}$. Since the transition points, $\mathrm{S}_{\mathrm{d}, \mathrm{DK}}$ and $\mathrm{S}_{\mathrm{d}, \mathrm{MM}}$, will be somewhat different for the two CLF models, a single measured value of the terminal time will lead to two different (model dependent) estimates for $\tau_{\mathrm{d}, 0}$ (and thus $\tau_{\mathrm{R}}$ ). To distinguish between the various estimates of these time constants we will adopt the following notation: $\left(\tau_{\mathrm{d}, 0}\right)_{\mathrm{DEMG}}$ and $\left(\tau_{\mathrm{R}}\right)_{\mathrm{DEMG}}$ for the values corresponding to the basic DE MG (or DE) model; $\left(\tau_{\mathrm{d}, 0}\right)_{\mathrm{MM}}$ and $\left(\tau_{R}\right)_{M M}$ for the values when the Milner-McLeish model for CLF is used, and $\left(\tau_{\mathrm{d}, 0}\right)_{\mathrm{DK}}$ and $\left(\tau_{\mathrm{R}}\right)_{\mathrm{DK}}$ when the Doi-Kuzuu version of CLF is used.

Finally, for a model that included DCR, the difference between the measured value $\tau_{\text {term }}$ and the estimate for $\tau_{\mathrm{d}, 0}$ (and thus $\tau_{\mathrm{R}}$ ) will be larger due to another "smaller than 1" factor on the right-hand side of eq 13 or eq 18. The value estimated for $\tau_{\mathrm{d}, 0}$ by fit to a model that contains only reptation will be smaller than that obtained using a model that contains CLF, and the latter will in turn be smaller than that obtained using a model that contains both CLF and DCR. When linear viscoelastic data do not extend to high enough frequencies to all ow a direct esti mate of $\tau_{R}$, it is clearly essential that estimates of relaxation times be derived from the same models that will be used for calculations in the nonlinear regime Consequently, we have elected to determine relaxation times for our model simulations directly from the linear viscoelastic data in Figures 1-5 using the same reptation models we will use when comparing with extensional flow data. We believe that this procedure provides more reliable values for $\tau_{\mathrm{R}}$ and $\tau_{\mathrm{d}, 0}$ than can be obtained from any current literature correlations.

Thus, the data in Figures $1-5$ were fit at low frequency using the linear viscoel astic limit of eq 10 to obtain the relaxation modulus for the whole chain [by integrating $\mathrm{G}\left(\mathrm{s}_{0}, \mathrm{t}, \mathrm{t}^{\prime}\right)$ over the unperturbed $\mathrm{s}_{0}$ from 0 to $\mathrm{L}_{0} / 2$ and multiplying by $\left.2 \mathrm{G}_{\mathrm{N}}^{0}\right]$, and then calculating $\mathrm{G}^{\prime}$ and $\mathrm{G}^{\prime \prime}$ via the standard formulas:

$$
\begin{aligned}
\mathrm{G}^{\prime}=\int_{0}^{\infty} \omega \mathrm{G}(\mathrm{t}) \sin \omega \mathrm{t} d \mathrm{t} \text { and } \\
\qquad \mathrm{G}^{\prime \prime}=\int_{0}^{\infty} \omega \mathrm{G}(\mathrm{t}) \cos \omega \mathrm{t} d \mathrm{t}
\end{aligned}
$$

These predictions are then compared with the linear viscoelastic data and adjustments are made in the estimated time constants until a best fit is achieved for each case [i.e., with the last term in eq 10 neglected or approximated by eq 11 or eq 14 depending on the model chosen]. The resulting predictions of the linear viscoelastic data are shown for the DE/DEMG model with no contour length fluctuations, and for the same model now including either the Milner-McLeish or the DoiKuzuu version of CLF. It can be seen that the qualitative correspondence between the DEMG/CLF models and the data is diminished beyond the crossing point for $\mathrm{G}^{\prime}$ and $\mathrm{G}^{\prime \prime}$. We shall see shortly that this is primarily because, in using eq 10 to calculate the relaxation modulus, we neglect diffusive constraint release (DCR) 49 and also neglect the higher order Rouse modes, which turn out to play a significant role at frequencies above the crossover frequency. The parameters $Z$ and $G_{N}^{0}$ were held fixed at the values quoted in Table 1.

One obvious question is what criteria to use in establishing a "best fit" for data over a limited range of frequencies. In the present study, we emphasize the importance of a good fit for $\mathrm{G}^{\prime \prime}$, which makes sense in view of the more limited accuracy of the $\mathrm{G}^{\prime}$ data at low frequencies where measurement limitations are relevant. It may be noted that a reasonably good first estimate of the terminal time can be obtained from the independently measured value of the zero shear viscosity (Table 1), via the approximate relationship:

$$
\eta_{0} \approx \mathrm{G}_{\mathrm{N}}^{0} \frac{\pi^{2}}{12} \tau_{\text {term }}
$$

With $\eta_{0}$ measured, and recalling that $\tau_{\text {term }}$ is interpreted as either $\tau_{\mathrm{d}, \mathrm{Mm}}$ or $\tau_{\mathrm{d}, \mathrm{DK}}$ for the present model, the approximations in eqs 12 and 13 or 18 and 19 may then be used to provide a first estimate of $\tau_{\mathrm{d}, 0}$.

The resulting estimates of the relaxation times $\left(\tau_{\mathrm{R}}\right)_{\mathrm{MM}}$, $\left(\tau_{\mathrm{d}, 0}\right)_{\mathrm{MM}},\left(\tau_{\mathrm{R}}\right)_{\mathrm{DK}}$, and $\left(\tau_{\mathrm{d}, 0}\right)_{\mathrm{DK}}$ are given in Table 2 . Also shown is the estimate of $\tau_{\text {term }}$ and the corresponding estimates of $\left(\tau_{\mathrm{R}}\right)_{\mathrm{DEMG}}$ and $\left(\tau_{\mathrm{d}, 0}\right)_{\text {DEMG }}$ for the DEMG model without any form of CLF. As explained above, $\tau_{\text {term }}=$ $\left(\tau_{\mathrm{d}, 0}\right)_{\text {DEMG }}$ for this case. We note that the estimated relaxation times for the Milner-McLeish version of CLF are actually closer to the DE/DEMG values than the predictions from the Doi-Kuzuu model. This result may seem surprising, as the Milner-McLeish model was developed to allow for faster relaxation near the chain ends where the entropic penalty for retraction does not yet play a significant role. A comparison of the fluctuation time scales as a function of position along the chain is shown in Figure 6 , based on the predicted time constants for the $3.9 \mathrm{M}, 10 \mathrm{wt} \%$ solution. Though the short-time process is clearly much faster for MilnerMcLeish, it is restricted to the region very near the end of the chain. Elsewhere, the roles are reversed, accounting for the larger estimates of the "bare" reptation time scale for the Doi-Kuzuu model.

4.3. A Comparison with Time Scale Estimates Using the Dedicated Linear Viscoelastic Models. An obvious question is how the estimated time scales obtained using our full model compare with estimates obtained using models that are derived specifically for 
Table 2. Relaxation Times

\begin{tabular}{|c|c|c|c|c|c|c|c|c|c|c|c|}
\hline $\begin{array}{l}\text { solution } \\
\text { name }\end{array}$ & $\begin{array}{c}\tau \text { term } \\
(\mathrm{s})\end{array}$ & $\begin{array}{c}\left(\tau_{\mathrm{d}, 0}\right)_{\text {DEMG }} \\
(\mathrm{s})\end{array}$ & $\begin{array}{l}\left(\tau_{\mathrm{R}}\right)_{\mathrm{DEMG}} \\
(\mathrm{s})\end{array}$ & $\begin{array}{c}\left(\tau_{\mathrm{d}, 0}\right)_{\mathrm{DK}} \\
(\mathrm{s})\end{array}$ & $\begin{array}{c}\left(\tau_{\mathrm{R}}\right)_{\mathrm{DK}} \\
(\mathrm{s})\end{array}$ & $\begin{array}{c}\left(\tau_{\mathrm{d}, 0}\right)_{\mathrm{MM}} \\
(\mathrm{s})\end{array}$ & $\begin{array}{c}\left(\tau_{\mathrm{R}}\right)_{\mathrm{MM}} \\
(\mathrm{s})\end{array}$ & $\begin{array}{c}\left(\tau_{\mathrm{d}, 0}\right)_{\mathrm{DE}}^{\mathrm{LV}} \\
(\mathrm{s})\end{array}$ & $\begin{array}{c}\left(\tau_{\mathrm{d}, 0}\right)_{\mathrm{MM}}^{\mathrm{LV}} \\
(\mathrm{s})\end{array}$ & $\begin{array}{c}\left(\tau_{\mathrm{d}, 0}\right)_{\mathrm{MM}}^{\mathrm{LM}} \\
(\mathrm{s})\end{array}$ & $\begin{array}{c}\left(\tau_{\mathrm{d}, 0}\right)_{\mathrm{DCR}}^{\mathrm{LM}} \\
(\mathrm{s})\end{array}$ \\
\hline $3.9 \mathrm{M}, 4.9 \mathrm{wt} \%$ & 4.56 & 4.56 & 0.23 & 43.3 & 2.19 & 19.1 & 0.97 & 4.31 & 11.1 & 39.4 & 113 \\
\hline $3.9 \mathrm{M}$ & 1.2 & 1. & 27 & 5.56 & 0.125 & 3.22 & 0.0 & 1. & 2.94 & 6.96 & 19.1 \\
\hline $3.9 \mathrm{M}, 10.0$ wt \% & 4.10 & 4.10 & 0.05 & 12.2 & 0.148 & 8.43 & 0.103 & 4.07 & 8.61 & 14.1 & 36.9 \\
\hline $3.9, M, 15.0$ wt $\%$ & 21.1 & 21.1 & 0.17 & 50.5 & 0.41 & 37.9 & 0.31 & 21.2 & 39.8 & 58.4 & 151. \\
\hline $10.2 \mathrm{M}, 6.0$ wt $\%$ & 39.6 & 39.6 & 0.51 & 123. & 1.58 & 83.4 & 1.08 & 39.1 & 83.7 & 201 & 467.2 \\
\hline
\end{tabular}

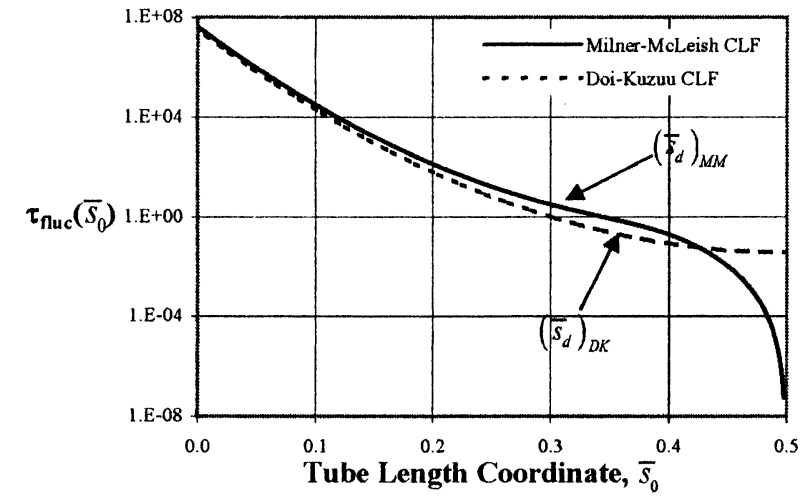

Figure 6. Fluctuation time scales as a function of position al ong the chain based on the predicted time scales for the 3.9 M 10.0 wt \% solution for the DE/Milner-MCLeish CLF (-) and DE/Doi-Kuzuu CLF (- - -) models.

the linear viscoelastic limit, such as the model of Milner and MCLeish ${ }^{15}$ or the more recent work of Likhtman and MCLeish. 49

There is not an obvious analytic way to reduce the full, coupled model used here [i.e., eqs 10 and 14-16] to the form of these linear viscoelastic models. On the other hand, Likhtman and McLeish have generously provided early access to a version of their code that incorporates an approximate version of the MilnerMcLeish model of CLF [eqs 14-16] and Rouse modes (but excludes DCR). We have used this code to fit the linear viscoelastic data with the same values of $\mathrm{G}_{\mathrm{N}}^{0}$ and Z. Clearly, since it incorporates eqs 14-16, we should expect it to yiel d estimates for the relaxation times that are similar to those obtained using the full model as described above. The results are shown in Table 2 for both the DE model (without CLF), labeled as $\left(\tau_{\mathrm{d}, 0}\right)_{\mathrm{DE}}^{\mathrm{LV}}$, and for the same model with Milner-McLeish CLF, denoted as $\left(\tau_{\mathrm{d}, 0}\right)_{\mathrm{MM}}^{\mathrm{LV}}$. The main point here is that the two sets of results, with the exception of the MilnerMcLeish estimate for the $4.9 \%$ solution, are in close agreement as expected.

Likhtman and McLeish have also used their more recent code $^{49}$ (using a more exact version of MilnerMCLeish CLF, both with and without DCR) to provide us with time scale estimates from our linear viscoelastic data, for the same fixed values of $G_{N}^{0}$ and $Z$. These linear viscoelastic fits have been carried out using a least squares criterion with equal weighting of the error in a log-log plot over the measured range of $\omega$. The resulting estimates for the relaxation times are shown in Table 2. The estimate induding Milner-McLeish CLF but without DCR is denoted as $\left(\tau_{d, 0}\right)_{M M}^{L M}$, while the result including DCR is denoted as $\left(\tau_{\mathrm{d}, 0}\right)_{\mathrm{DCR}}^{\mathrm{LM}}$. With the change to a more exact version of Milner-McLeish theory and the change in weighting of the errors, the estimate of $\tau_{\mathrm{d}, 0}$ is larger than the value we obtained using the approximate Milner-Mdeish theory $\left[\left(\tau_{\mathrm{d}, 0}\right)_{\mathrm{MM}}\right]$. I ronically, it is actually closer to the value we obtained using the Doi-Kuzuu version of CLF $\left[\left(\tau_{\mathrm{d}, 0}\right)_{\mathrm{DK}}\right]$. When
DCR is added, the estimates of $\tau_{\mathrm{d}, 0}$ increase by a factor of 2-3 for reasons discussed earlier. Since the nonlinear models [eqs 10, 14-16] do not include DCR, we provide these results primarily for future reference.

Finally, returning to Figures $1-5$, we see that the theor etical predictions using the models from eq 10 with their appropriate estimates of relaxation time scales agree reasonably well with experimental data in the fitted region (i.e., in the region prior to the crossing point for $G^{\prime}$ and $\left.G^{\prime \prime}\right)$, but differ beyond the crossing point. The most obvious exceptions are the $3.9 \mathrm{M}, 4.9 \mathrm{wt} \%$ solution, where there is evidently some problem with the $\mathrm{G}^{\prime \prime}$ data at the lowest frequencies, and the $10.2 \mathrm{M}, 6.0$ wt \% solution, which is almost certainly affected by the higher polydispersity of the polymer coupled with the absence of diffusive constraint release in the model.

As noted above, the major differences between the predictions from the nonlinear models and the experimental data are in the qualitative shape of the curves for the regime beyond the crossing point of $\mathrm{G}^{\prime}$ and $\mathrm{G}^{\prime \prime}$. However, this is not a reflection of the time scale estimates, but due to the fact that the nonlinear model given by eqs 10 and 14-16 neglects the contributions made by Rouse modes and the DCR mechanism, which are important in this region. To illustrate this point, we have included a plot in Figure $3 b$ of the predicted results for the $3.9 \mathrm{M}, 10.0 \mathrm{wt} \%$ solution for several different versions of the specialized linear viscoelastic codes. Two predictions come from the approximate Milner-McLeish code described above, one with Milner-MCLeish CLF and no Rouse modes and the other with Milner-McLeish CLF including the Rouse modes. When we include Milner-MCLeish CLF and no Rouse modes, we obtain results that are similar to those obtained previously with our nonlinear model also including Milner-McLeish CLF. We have al ready noted that the time constants for these two cases are nearly identical [i.e., $\left(\tau_{\mathrm{d}, 0}\right)_{\mathrm{MM}}$ and $\left(\tau_{\mathrm{d}, 0}\right)_{\mathrm{MM}}^{\mathrm{LV}}$ ]. However, when we include Rouse modes using exactly the same time constant, $\left(\tau_{\mathrm{d}, 0}\right)_{\mathrm{MM}}^{\mathrm{LV}}$, the contribution is significant even at these relatively low frequencies, and the prediction for $\mathrm{G}^{\prime}$ looks much better. The second pair of results shown in Figure $3 \mathrm{~b}$ are predictions from the newer model of Likhtman and MCLeish, including CLF and Rouse modes in both cases but excluding DCR in one case and including it in the other. We see that DCR adds a significant upward shift in $\mathrm{G}^{\prime}$ beyond the crossing point. While the result including DCR does not look much different than that obtained using the MilnerMCL eish model without DCR, we should remember that the time constant estimates and the treatment of CLF are different in the two cases.

We emphasize that the nonlinear models used in the next section for comparisons with the nonlinear extensional flow data contain an approximation of the Rouse modes in the form of the chain stretching model but do not contain DCR. Whether the neglect of DCR and the approximation of the Rouse behavior is a significant problem in the nonlinear flow regimeremains to beseen. 
We see, however, that the estimated time constants from our model are essentially equal to those from a dedicated linear viscoelastic model that treats MilnerMCLeish CLF in the same approximate way, and that a model that includes Rouse modes and/or DCR gives reasonable predictions of the linear viscoelastic data over the whole measured range.

We note that even when all of the mechanisms are included in the linear viscoelastic flow models, fits to the linear viscoelastic data are still imperfect. The magnitude of the peak and/or the depth of the predicted minimum following the crossover of $\mathrm{G}^{\prime}$ and $\mathrm{G}^{\prime \prime}$ are both larger in the predictions than in the data. In contrast, linear viscoelastic data for polymer melts with a similar degree of chain entanglement show a much more pronounced peak in $\mathrm{G}^{\prime \prime}$ and a slope after the peak that varies between $-1 / 2$ and $-1 / 4^{9}$ that is similar to the model predictions. One possible contributor to the flattening of the plateau region in the solution data is the polydispersity of the polymer samples. Indeed, this point is clear if we compare the results in Figure 5 with those from Figure 3. On the other hand, the data presented here for the low polydispersity 3.9 M molecular weight polystyrene solutions is typical of the vast majority of solution data in the literature in the sense that, for frequencies above the crossover frequency, G" exhibits a very shallow minimum at best, even for nearly monodisperse polymers. These factors suggest that there may be a more fundamental and subtle difference between entangled solutions and melts associated with the fact that the molecular weight between entanglements is much larger when the polymer chains are immersed in a solvent. Such distinctions have not yet been accounted for theoretically. We believe that this is an important issue for future research, and we are aware of ongoing work by McLeish that was motivated by the results in Figures $1-5$ and their similarity to other "monodisperse" solution data.

\section{Steady State Results for Uniaxial Extensional Flow}

Now that we have determined model parameters, we are in a position to make comparisons with experimental data in the nonlinear regime. We begin with steady state results for uniaxial extensional flow. Our presentation will be split into three parts. First, in section 5.1, we present and discuss the data. In section 5.2, we then present and discuss model predictions without commenting specifically on the comparison with data. We do this because it is ultimately helpful to first understand the differences between the predictions of the various models. Finally, in section 5.3, we discuss the comparisons between the data and the model predictions.

5.1. Experimental Data for Steady Flow. The principal experimental results from this study are shown in Figures 7 and 9-12, where we plot the extensional viscosity, $\eta_{\mathrm{e}}$, as a function of the uniaxial rate of strain $\dot{\epsilon}\left(\equiv \partial \mathrm{v}_{\mathrm{z}} / \partial \mathrm{z}\right)$ for steady uniaxial extensional flow. Also shown in these figures are predictions from various models. In this section, we focus exclusively on the experimental data.

The extensional viscosity is the ratio of the measured normal stress difference, $\sigma_{\mathrm{zz}}-\sigma_{\mathrm{rr}}$, to $\dot{\epsilon}$. We use the actual strain rates rather than a dimensionless strain rate such as the Weissenberg number based on the Rouse time (e.g., $\mathrm{Wi}_{\mathrm{R}} \equiv \dot{\epsilon} \tau_{\mathrm{R}}$ ), because we ultimately want to
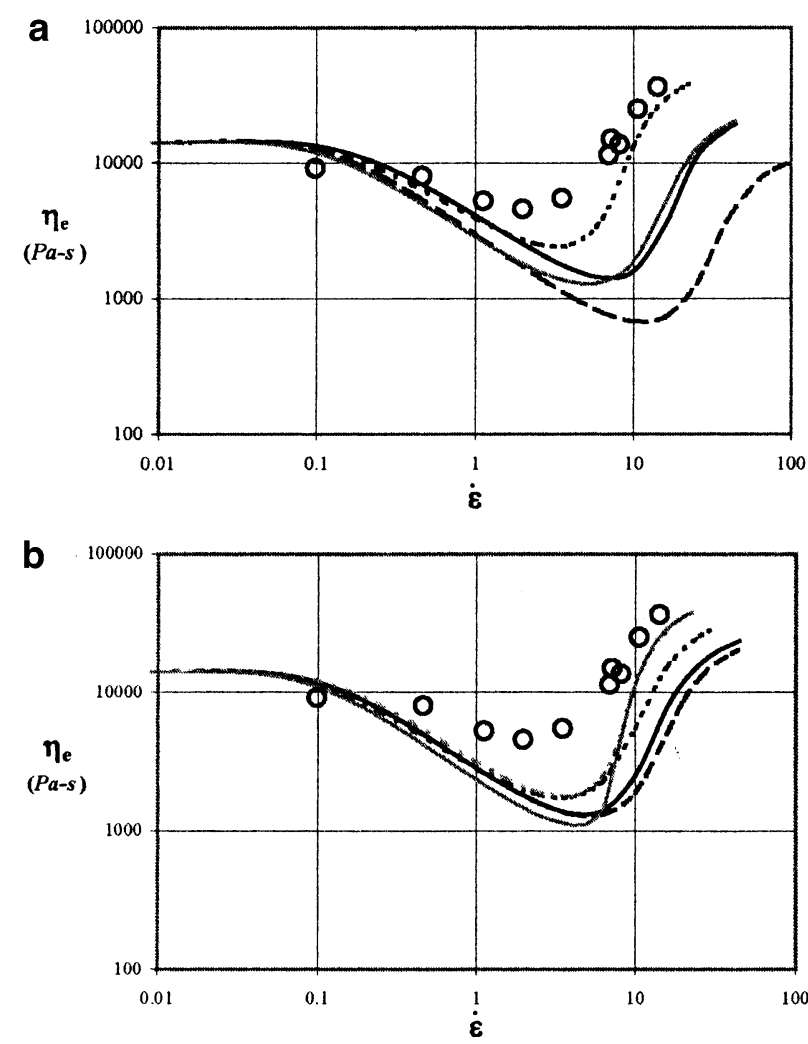

Figure 7. (a) Extensional viscosity as a function of strain rate for the $3.9 \mathrm{M} 10.0 \mathrm{wt} \%$ solution: $(O)$ experimental data, $(-)$ basic DEMG model, (- -) MLD model, (- - ) DEMG/MilnerMCLeish CLF model, and (gray line) MLD/Milner-McLeish CLF model. (b) Extensional viscosity as a function of strain rate for the $3.9 \mathrm{M} 10.0 \mathrm{wt} \%$ solution: (O) experimental data, (-) MLD/Milner-McLeish CLF with ad hoc switch function, (- -) MLD/Milner-McLeish CLF model with self-consistent switch function, (- - ) MLD/Doi-Kuzuu CLF model with selfconsistent switch function, (gray line) Marrucci CCR $(\beta=3.6)$ / Milner-McLeish CLF model, and (gray hyphens) Marrucci CCR $(\beta=1.0) /$ Milner-McLeish CLF model.

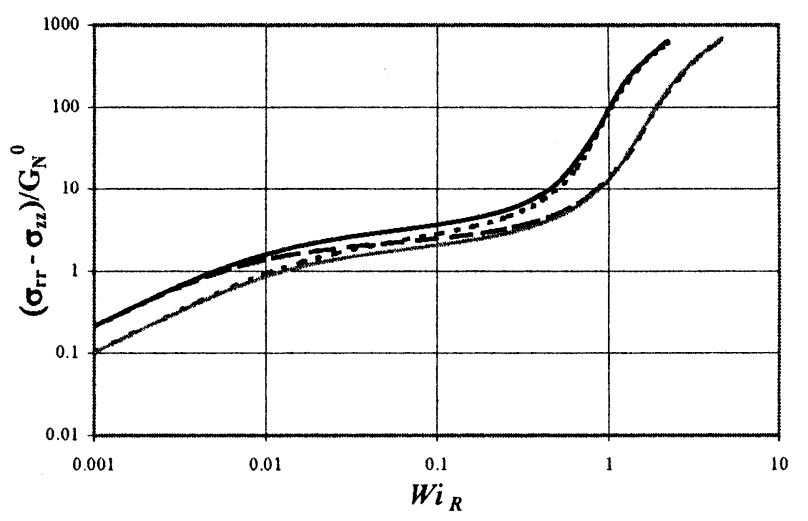

Figure 8. Dimensionless normal stress difference $\left(\sigma_{\mathrm{zz}}-\sigma_{\mathrm{rr}}\right)$ vs the Weissenberg number based on the Rouse times $\left(\mathrm{Wi}_{\mathrm{R}} \equiv\right.$ $\dot{\epsilon} \tau_{\mathrm{R}}$ ) for the same model predictions as in $\mathrm{F}$ igure $7 \mathrm{a}$.

make comparisons with both DEMG models and models that contain CLF. Since the appropriate estimate for $\tau_{\mathrm{R}}$ depends on the choice of models, the experimental data would shift for each model if plotted $\mathrm{vS} \mathrm{Wi}_{\mathrm{R}}$ rather than $\dot{\epsilon}$. We note that the practice of determining relaxation time scales independently for each version of the model based on the low frequency viscoelastic data (or alternatively, $\eta_{0}$ ) requires the model predictions for the extensional viscosity to yield the expected Trouton ratio of $3 \eta_{0}$ in the limit $\dot{\epsilon} \rightarrow 0$. 


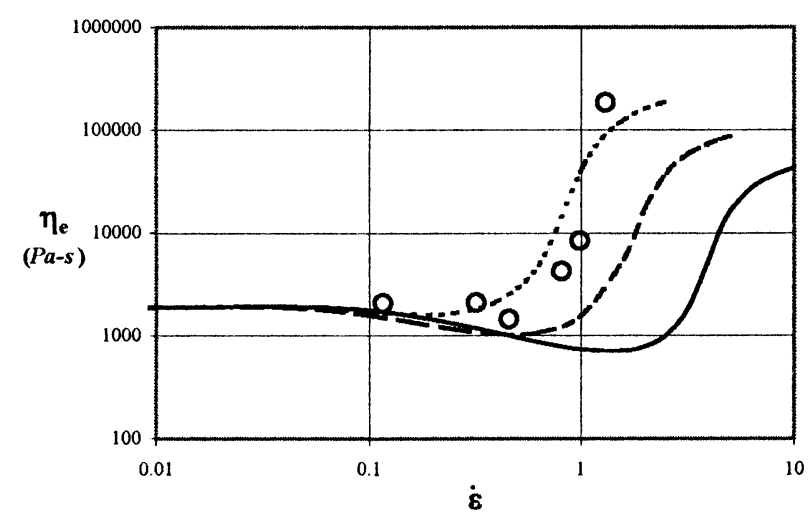

Figure 9. Extensional viscosity as a function of the strain rate for the $3.9 \mathrm{M} 4.9 \mathrm{wt} \%$ solution: (O) experimental data, $(-)$ basic DEMG model, $(--)$ MLD/Milner-McLeish CLF model, and (- - ) MLD/Doi-Kuzuu CLF model.

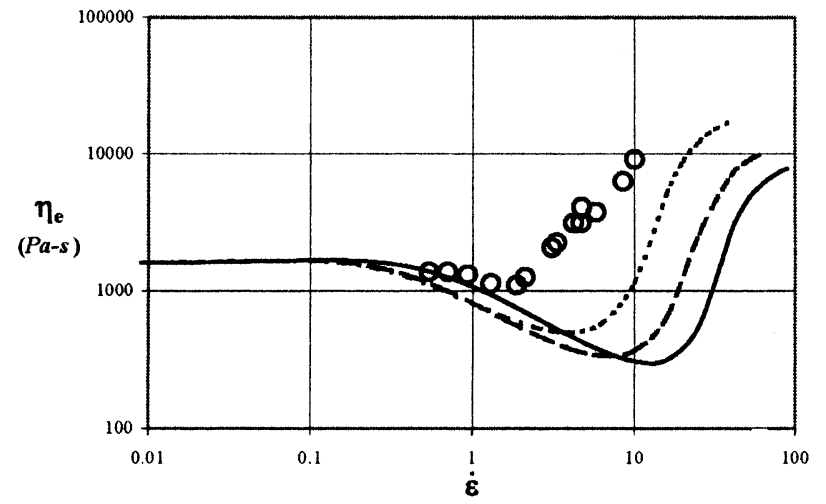

Figure 10. Extensional viscosity as a function of the strain rate for the $3.9 \mathrm{M} 7.35 \mathrm{wt} \%$ solution: (O) experimental data, $(-)$ basic DEMG model, $(--)$ MLD/Milner-McLeish CLF model, and (- - -) MLD/Doi-Kuzuu CLF model.

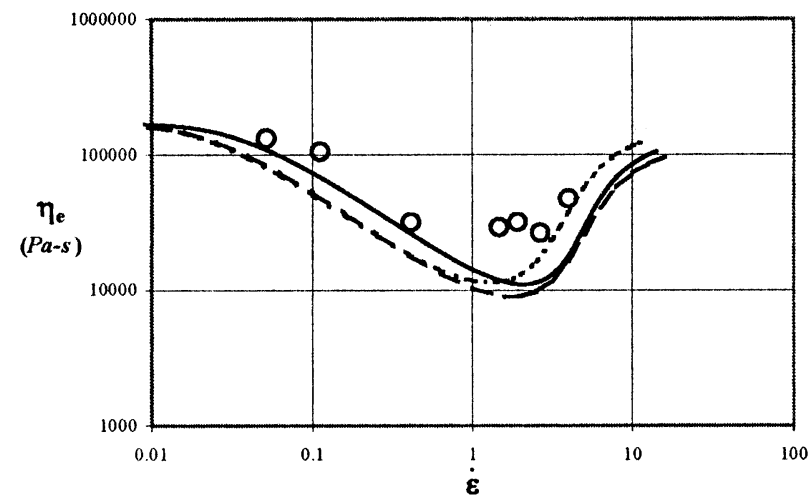

Figure 11. Extensional viscosity as a function of the strain rate for the $3.9 \mathrm{M} 15.0$ wt \% solution: (O) experimental data, (-) basic DEMG model, (- -) MLD/Milner-McLeish CLF model, and (- - ) MLD/Doi-Kuzuu CLF model.

Examining the data in Figures 7 and 9-12, we see that as the strain rate is increased, the extensional viscosity at first decreases. Qualitatively, this behavior is similar to that seen in earlier rheo-optical experiments. ${ }^{37-39,64,65}$ In the present data, however, when the strain rate is increased to a value on the order of the inverse Rouse time [i.e., $\mathrm{Wi}_{\mathrm{R}} \sim \mathrm{O}(1)$ ], there is a relatively sharp upturn in the extensional viscosity reflecting the onset of chain stretching. While chain stretching is expected, it has not previously been experimentally demonstrated with this degree of clarity. In the earlier rheo-optical studies, the flow cell was either a two- or

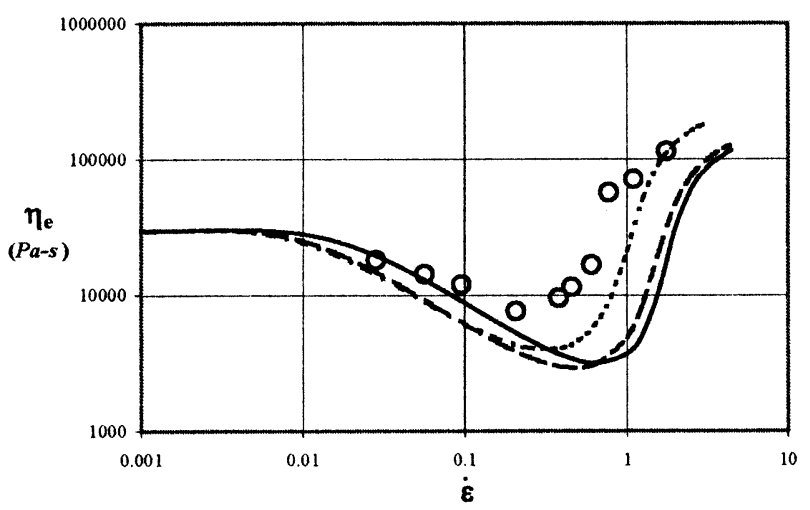

Figure 12. Extensional viscosity as a function of the strain rate for the $10.2 \mathrm{M} 6.0 \mathrm{wt} \%$ solution: (O) experimental data, (-) basic DEMG model, (--) MLD/Milner-McLeish CLF model, and (- - ) MLD/Doi-Kuzuu CLF model.

four-roll mill, and the shear-thinning nature of the flow in the immediate vicinity of the rollers made it difficult to achieve the high strain rates at the central stagnation point necessary to demonstrate the upturn in the extensional stress so convincingly.

The data for the lowest concentration solutions (3.9 $\mathrm{M}, 4.9$ wt \% and $3.9 \mathrm{M}, 7.35$ wt \%), shown in Figures 9 and 10 , exhibit very little strain rate thinning prior to the upturn in viscosity. It is known that the extensional viscosity for dilute solutions of unentangled, linear, flexible polymer increases monotonically from the limiting Trouton value of $3 \eta_{0}$ with increasing strain rate for $\dot{\epsilon} \tau_{\mathrm{R}}{ }^{-1} \geq \mathrm{O}(1)$. Thinning at lower values of the Weissenberg number is characteristic of polymeric liquids with a significant number of entanglements per chain. The presence of a large number of entangl ements leads to a separation of the relaxation time scale for orientation relative to that for chain stretch, which increases with $\mathrm{Z}$ as $\tau_{\mathrm{d}, 0} / \tau_{\mathrm{R}}=3 \mathrm{Z}$ as we have al ready discussed. For $\tau_{\mathrm{d}, 0^{-1}}$ $\leq \dot{\epsilon} \leq \tau_{\mathrm{R}}{ }^{-1}$, as $\dot{\epsilon}$ is increased, the polymer chains become increasingly aligned with the principal axis of strain, but they do not stretch, and the increase of $\sigma_{\mathrm{zz}}-\sigma_{\mathrm{rr}}$ is slower than the increase in strain rate, causing the extensional viscosity to decrease. Once chain stretching begins for $\dot{\epsilon} \tau_{\mathrm{R}}{ }^{-1} \geq \mathrm{O}(1)$, the extensional viscosity increases. As the number of entanglements per chain decreases, the strain rates corresponding to the onset of chain stretch and chain alignment become closer until they are equal in the dilute limit. It may at first seem surprising that the $3.9 \mathrm{M}, 7.35$ wt \% solution data does not show more strain rate thinning, as it has approximately 15 entanglements per chain and hence a large ratio of $\tau_{\mathrm{d}, \mathrm{o}} / \tau_{\mathrm{R}}$. However, it is really the ratio of the longest effective rel axati on time for chain alignment and the Rouse time, $\tau_{\text {term }} / \tau_{\mathrm{R}}$, that control s the separation in $\dot{\epsilon}$ between the onset of chain orientation and stretch, and the combined effects of CLF and DCR reduce this rati o to a rather small value for the $7.35 \mathrm{wt} \%$ solution.

Qualitatively, the degree of strain rate thinning increases and the amount of chain stretching decreases as the solutions become more entangled (holding the molecular weight constant), and the same is true if the number of entanglements per chain is held fixed and the molecular weight of the polymer is increased. In either case, the molecular weight between entanglements is the key parameter. From a scaling point of view, the maximum extension the polymer chain can undergo is equal to $\sqrt{\mathrm{N} / \mathrm{Z}}$, where $\mathrm{N}$ is the number of Kuhn steps and $Z$ is the number of entangl ements per 
chain. ${ }^{12,13}$ As the number of entanglements increases for fixed molecular weight or as the molecular weight is decreased for a fixed number of entanglements per chain, the molecular weight between entanglements decreases; consequently, the chain appears less extensible and the tendency of the chain to experience segmental stretching is diminished.

5.2. Steady Flow Predictions. We now consider the model predictions for this same steady flow, which are also plotted in Figures 7-12. In this section, we first present and compare the theoretical predictions for the various models. Comparison with data is then reserved until section 5.3.

The model predictions in Figures 7-12 were obtained using the values of $\tau_{\mathrm{d}, 0}, \tau_{\mathrm{R}}, \mathrm{Z}$, and $\mathrm{G}_{\mathrm{N}}^{0}$ from Tables 1 and 2 for each particular model. Specifically, for the DEMG model and the MLD model without CLF we use the estimates $\left(\tau_{\mathrm{d}, 0}\right)_{\mathrm{DEMG}}$ and $\left(\tau_{\mathrm{R}}\right)_{\mathrm{DEMG}}$, while for the models with CLF we use either $\left(\tau_{\mathrm{d}, 0}\right)_{\mathrm{DK}}$ and $\left(\tau_{\mathrm{R}}\right)_{\mathrm{DK}}$ or $\left(\tau_{\mathrm{d}, 0}\right)_{\mathrm{MM}}$ and $\left(\tau_{\mathrm{R}}\right)_{\mathrm{MM}}$.

The most complete set of results is shown in Figures 7 and 8 for the $3.9 \mathrm{M}, 10.0 \mathrm{wt} \%$ solution. Since the other solutions show similar behavior, we only show a reduced set of model calculations for those cases.

Let us begin with Figure $7 a$. The solid black line represents the basic DEMG predictions without either CCR or CLF. The long-dashed line below it is the result obtained using the Mead et al. (MLD) model for convective constraint rel ease (CCR) but without including any mechanism for contour length fluctuations (CLF). In these cases, since CCR does not effect the linear viscoelastic predictions of the model, the appropriate values of the relaxation times are the DEMG values. Hence, the only differences in the predicted behavior are a direct consequence of the addition of the CCR mechanism to the basic DEMG model. At low strain rates where CCR is insignificant, the two results are coincident, and both show that the onset of strain rate thinning occurs at a strain rate that is approximately $0.5\left(\tau_{\mathrm{d}, 0}\right)_{\text {DEMG }}$. However, the inclusion of CCR causes the extensional viscosity to decrease more rapidly with increasing strain rate. In addition, the upturn in $\eta_{\mathrm{e}}$, signifying the onset of chain stretch, is predicted to occur at a larger value of $\dot{\epsilon}$.

To understand these results, we might first ask why the extensi onal viscosity initially decreases with strain rate for the DEMG model. As the strain rate is increased above $\mathrm{O}\left(\tau_{\mathrm{d}}{ }^{-1}\right)$, the polymer chains begin to align with the principal strain rate axis, and thus the stress difference $\sigma_{\mathrm{zz}}-\sigma_{\mathrm{rr}}$ increases. However, the degree of alignment increases more slowly than the strain rate and eventually saturates, causing the stress to increase more slowly than the strain rate and the extensional viscosity to decrease. When CCR is included as an additional relaxation mechanism, the relaxation time for chain alignment decreases with increasing strain rate; hence, the degree of alignment is reduced, and the extensional viscosity decreases more rapidly than it does without CCR. This physical description should be contrasted with the effect of CCR on the shear stress (or shear viscosity) for simple shear flow. In that case, faster relaxation of segmental orientation again decreases the degree of alignment, but that leads to an increase in the shear stress at any given shear rate and a decrease in the rate of shear thinning. Finally, in the MLD model, there is a gradual transition from CCR causing faster relaxation of alignment to faster relax- ation of chain stretch when $\mathrm{Wi}_{\mathrm{R}} \geq \mathrm{O}(1)$. As a result, the onset of chain stretch is delayed to larger values of the strain rate, and the extensional viscosity curve is shifted to the right relative to the basic DEMG prediction.

The next mechanism to consider is CLF, and for this purpose it is useful to compare the prediction "DEMG/ Milner-McLeish CLF" in Figure 7a with the basic DEMG prediction. In the former, we include CLF but exclude any CCR contributions. When contour length fluctuations are incorporated into the DEMG model, they add another mechanism for the relaxation of chain alignment. One would again expect the extensional viscosity to shift to lower values when compared with the basic DEMG model. However, in Figure 7a, we see that the DEMG/Milner-McLeish CLF curve is actually shifted above the DEMG prediction rather than below it as "expected." To understand this result, we must remember that the estimated relaxation times $\left(\tau_{\mathrm{d}, 0}\right)_{\mathrm{MM}}$ and $\left(\tau_{\mathrm{R}}\right)_{\mathrm{MM}}$ are significantly larger than the estimated values for the DEMG model, $\left(\tau_{\mathrm{d}, 0}\right)_{\text {DEMG }}$ and $\left(\tau_{\mathrm{R}}\right)_{\mathrm{DEMG}}$. As a result, the predictions at any given strain rate shift to the left relative to those for the model without CLF. When CCR is added back into the model ("MLD/MilnerMcLeish CLF"), the predicted values are again shifted to lower $\eta_{\mathrm{e}}$, and the onset of chain stretch is delayed for exactly the same reason discussed in the preceding paragraph. It should be noted that the DEMG and MLD model s with Milner-McLeish CLF use the same relaxation times from Table 2, $\left(\tau_{\mathrm{d}, 0}\right)_{\mathrm{MM}}$ and $\left(\tau_{\mathrm{R}}\right)_{\mathrm{MM}}$. It is somewhat ironic that the full MLD/Milner-McLeish CLF model predictions are actually quite close to the predictions for the simple DEMG model without either CLF or CCR.

Finally, in Figure $7 \mathrm{~b}$, we show several additional results, including MLD/Milner-McLeish CLF but with the "ad hoc" form of the switch function $g$ [see eq 22], the MLD model with the Doi-Kuzuu version of CLF, and the DEMG model with a combination of MilnerMCLeish CLF and the Marrucci version of CCR. We will discuss the latter momentarily. Here, we note that there is relatively little difference between the exact and ad hoc switch functions, at least for this class of flows. Hence, in all of the predictions that we have made using the MLD version of CCR [including those in Figure 7a], we use the "self-consistent" rather than the "ad hoc" switch function. A more important comparison is that between the predictions of the "MLD/Doi-Kuzuu CLF" model [using the time constants $\left(\tau_{\mathrm{d}, 0}\right)_{\mathrm{DK}}$ and $\left(\tau_{\mathrm{R}}\right)_{\mathrm{DK}}$ ] and the "MLD/Milner-McLeish CLF" model [the same result as in Figure 7a with time constants $\left(\tau_{\mathrm{d}, 0}\right)_{\mathrm{MM}}$ and $\left.\left(\tau_{\mathrm{R}}\right)_{\mathrm{MM}}\right]$. Although these model s are equival ent in terms of fitting the linear viscoelastic data in the fitted region, there is a nontrivial difference between the predictions in nonlinear extensional flow, even though they differ only in the CLF part of the model.

We have noted previously that it is impractical to plot the data vs a dimensionless strain rate $\left(\mathrm{Wi}_{\mathrm{R}} \equiv \dot{\epsilon} \tau_{\mathrm{R}}\right)$, because the estimate of $\tau_{\mathrm{R}}$ is model dependent, and we would need to replot the data for each model comparison. However, we can reasonably plot the various model predictions versus $\mathrm{Wi}_{\mathrm{R}}$. Additionally, we can also plot the dimensionless stress $\left(\sigma_{\mathrm{zz}}-\sigma_{\mathrm{rr}}\right) / \mathrm{G}_{\mathrm{N}}^{0}$ rather than the extensional viscosity. These changes eliminate the effects of shifts in $\dot{\epsilon}$ due to changes in $\tau_{\mathrm{R}}$ and $\tau_{\mathrm{d}, 0}$, and thus allow a direct examination of the contributions of the various physical mechanisms. Hence, we replot the various results from Figure 7a in Figure 8 as $\left(\sigma_{\mathrm{zz}}-\sigma_{\mathrm{rr}}\right) /$ 
$\mathrm{G}_{\mathrm{N}}^{0}$ vs $\mathrm{Wi}_{\mathrm{R}}$ instead of $\eta_{\mathrm{e}}$ vs $\dot{\epsilon}$, so that the role of the various mechanisms is clearer. Beginning with the results for DEMG, we see that as we add either CLF or CCR alone, we shift the extensional stress to lower values. For CLF, the shift appears for small values of $\mathrm{Wi}_{R}$, while for CCR it occurs for large values of $\mathrm{Wi}_{R}$. When both mechanisms are included (MLD/MilnerMCLeish CLF), the net effect is additive, shifting the stress to lower values for all $\mathrm{Wi}_{\mathrm{R}}$.

Finally, as noted above, Figure $7 b$ shows two sets of predictions for the simpler Marrucci CCR model. One of these uses the parameter value of $\beta=3.6$ that was shown in an earlier study to provide the best fit for shear flow data ${ }^{38}$ when combined with the MilnerMCLeish version of CLF. The second Marrucci CCR prediction uses a smaller value of the parameter, $\beta=$ 1. As expected, reducing $\beta$ diminishes the effect that CCR has on chain alignment, resulting in $\eta_{\mathrm{e}}$ shifting closer to the DEMG/Milner-McLeish CLF prediction for strain rates less than about $0.1 \mathrm{~s}^{-1}$. One fundamental difference between the Marrucci and MLD models for CCR is that the former assumes that the CCR mechanism only affects the relaxation of chain alignment and has no direct effect on chain stretch, whereas the MLD model hypothesizes a transition from enhanced relaxation of orientation to faster relaxation of chain stretch. As a result, when $\mathrm{Wi}_{\mathrm{R}} \geq \mathrm{O}(1)$, the Marrucci CCR predictions in Figure $7 \mathrm{~b}$ merge with the results for the DEMG/Milner-McLeish CLF model [Figure 7a] without CCR.

Theoretical predictions for the other solutions are shown in Figures 9-12 and are limited to basic DEMG and the full MLD model that includes either the DoiKuzuu or Milner-McLeish version of CLF. Because the other results are qualitatively similar to those discussed above in relation to Figure 7 and in accord with expectations, we believe that there is little to be learned by continuing to show calculations for all possible combinations of models. The theoretical predictions in Figures 9-12 confirm our expectations that as the number of entanglements per chain is reduced and the separation between $\tau_{\mathrm{R}}$ and $\tau_{\mathrm{d}, 0}$ is reduced, the amount of strain rate thinning diminishes while the degree of chain stretching increases as the ratio $\mathrm{N} / \mathrm{Z}$ increases. These trends are particularly evident in comparing Figures 7 and 12, where the number of entanglements per chain is the same, but the ratio N/Z increases by a factor of 2.6. The predicted degree of thinning with increasing strain rate is nearly identical, but there is clearly a larger degree of chain stretch.

We note that the distinction between the basic DE MG model and the two versions of MLD increases as Z decreases. This is to be expected, since CLF plays an increasingly important role in the relaxation process for lightly entangled chains. However, for the three cases with the largest number of entanglements per chain (see Figures 7, 11, and 12), the distinction between the MLD model and the DE MG model is relatively weak. As noted earlier, the corrections to DEMG due to CLF and CCR largely cancel out when compared with relaxation times that give equivalent fits to the linear viscoelastic data.

5.3. Comparison with Experimental Data. Wecan now compare the model predictions with the experimental data. From a qualitative point of view, the comparison is favorable, especially for the models with CLF included. However, quantitatively, the model predictions are uniformly low over the whole range of measured strain rates. The differences decrease as the number of entanglements per chain is increased, but this is largely because the simple DE MG model does a better job in this regime and the corrections due to CLF and CCR become less important. We have noted in the previous section that the two versions of CLF give significantly different results in the nonlinear regime, though they are equivalent in the linear viscoelastic limit. Of the two, it appears that the Doi-Kuzuu CLF model better represents the data. Indeed, on the basis of the results in this section, if one were to simulate an extensional flow with any of the models that we have investigated, the MLD model with Doi-Kuzuu CLF would seem to be the best choice provided one had linear viscoelastic data to determine the relaxation times for this model.

This does not mean, however, that the Doi-Kuzuu model is a fundamentally better model for CLF than Milner-McLeish. In fact, on the basis of the work of Milner and MCLeish ${ }^{15}$ and Lihktman and MCLeish, 49 one would believe that this is not the case. The point to keep in mind is that DCR has still not been considered in these models. A comparison of $\left(\tau_{\mathrm{d}, 0}\right)_{\mathrm{MM}}^{\mathrm{LM}}$ and $\left(\tau_{\mathrm{d}, 0}\right)_{\mathrm{DCR}}^{\mathrm{LM}}$ derived in section 4.2 from our linear viscoelastic data using the model of Lihktman and MCLeish, ${ }^{49}$ the first with CLF and the second with CLF and DCR, suggests that the bare reptation time $\tau_{\mathrm{d}, 0}$ (and thus the estimate of $\tau_{\mathrm{R}}$ as well) will be reduced by a factor of between 2 and 3 once DCR is added to the nonlinear model. This will improve the data fits in the chain stretching regime, as it will shift the predictions to the left relative to the data.

The disagreement between data and predictions is not just a matter of shifting the time scales, however. For the models considered here, the depth of the minimum in the viscosity is also too large. Although this result would likely be improved when DCR is added to the model, it has recently been suggested ${ }^{66-69}$ that the simple CCR mechanisms of Marrucci and co-workers ${ }^{19-21}$ as well as the CCR mechanism in the MLD model ${ }^{1}$ are also likely to underestimate the effect of the convective constraint release process. Specifically, to account for the fact that each constraint release event involves a pair of chains, it has been proposed to model the CCR process as a "double reptation" event. The combination of DCR and a dual release CCR will certainly change the shapes of the curves; thus, it is premature to speculate further about whether a moreadvanced model of the MLD form will ultimately provide quantitative predictions for extensional flow data. Comparisons between the data reported here and model predictions that take account of these ideas are currently in progress, but the requisite devel opment of the nonlinear models is not available at the present time.

Returning to Figures 7 and 9-12, it may seem surprising at first to note that the most important differences between the various models seem to appear in the high strain rate, chain stretching regime, although both CLF and CCR were originally added to the DE/DEMG model to correct predictions for shear flow in the low strain rate regime of validity of the original $\mathrm{DE}$ theory [i.e., $\dot{\epsilon} \leq \mathrm{O}\left(\tau_{\mathrm{R}}{ }^{-1}\right)$ ]. One might think that, regardless of what is done to the model in this regime, the model dynamics should be dominated by the chain stretching mechanism (and higher order Rouse modes) at strain rates in excess of $\tau_{\mathrm{R}}{ }^{-1}$. Thus, one would expect model predictions to be quite similar in this regime, 
which is clearly not the case. A primary culprit is the model dependence of relaxation times. As we add mechanisms that influence the dynamics at low frequency, we have seen that this changes the estimated value of the "bare" reptation time obtained from the longest measurable relaxation time, which in turn changes the estimated Rouse time. One could argue that if we had an independent way to determine the Rouse time, $\tau_{R}$, then we should use the same Rouse time for all models. Unfortunately, unless the model is "complete," fixing a Rouse time and maintaining the expected ratio of $\tau_{\mathrm{d}, 0} / \tau_{\mathrm{R}}$ would then change all of the predictions at low strain rate, including the zero shear viscosity that would then become model dependent.

Finally, we observe that the comparison between the theory and experiment is least satisfactory for the 3.9 M, 7.35 wt \% solution, the results of which are shown in Figure 10. We cannot offer an explanation for this. Of course, the number of entanglements per chain is smaller in this case than in those shown in Figures 7 , 11 , and 12 , but it is still large enough that we would have expected better agreement between the theory and the experiment. One satisfactory feature of the results shown in Figures 9 and 10 is that the theory does capture the qualitative feature of a transition from entangled to "dilute" behavior. In particular, for the lowest concentration at 4.9 wt \%, both the theory and the experiment indicate that the extensional viscosity increases at the onset of chain stretching without any prior thinning.

\section{Transient Flow Data}

In addition to the steady flow data presented above, we have also obtained data for stress growth upon startup of the extensional flow from rest. Typical data are shown in Figure 13, parts a-c, for the $3.9 \mathrm{M}, 10.0$ wt \% solution at three different values of the strain rate, $\dot{\epsilon}=1.12,3.49$, and $7.19 \mathrm{~s}^{-1}$. We have chosen to plot these data in terms of an "extensional viscosity" in order to provide a direct comparison with the steady state results presented above, though it is well-known that the concept of an extensional viscosity is ambiguous in transient flow conditions. The "extensional viscosity" reported here is simply the instantaneous extensional stress difference $\left(\sigma_{\mathrm{zz}}-\sigma_{\mathrm{rr}}\right)$ scal ed by the instantaneous strain rate. Since the latter is constant and independent of time, the results are equival ent to plotting the stress difference vs time. For all three strain rates, the extensional viscosity grows monotonically to the steadystate values reported in Figure 7. The total strain, $\dot{\epsilon}$ t, for $\dot{\epsilon}=1.12 \mathrm{~s}^{-1}$ is 2.24 , for $\dot{\epsilon}=3.49 \mathrm{~s}^{-1}$ it is 5.24 , and for $7.19 \mathrm{~s}^{-1}$ it is 6.83 . The latter two values are well into the steady-state regime, while the value at $\dot{\epsilon}=1.12$ $\mathrm{s}^{-1}$ appears to be marginal .

Figure 13 also shows predictions for three models: the basic DEMG model using $\left(\tau_{\mathrm{R}}\right)_{\mathrm{DEMG}}$ and $\left(\tau_{\mathrm{d}, 0}\right)_{\mathrm{DEMG}}$; and the full MLD model with, respectively, the MilnerMCLeish version of CLF using $\left(\tau_{R}\right)_{M M}$ and $\left(\tau_{d, 0}\right)_{M M}$, and the Doi-Kuzuu version of CLF using $\left(\tau_{\mathrm{R}}\right)_{\mathrm{DK}}$ and $\left(\tau_{\mathrm{d}, 0}\right)_{\mathrm{DK}}$. Since the predicted response to the startup flow is a monotonic rise from the initial value of zero to the final steady-state value for all versions of the model, there is not much to be learned beyond what we already observed in Figure 7 as far as the magnitudes of $\eta_{\mathrm{e}}$ are concerned.

Of more interest and importance is the qualitative shape of the predictions and particularly the time scale
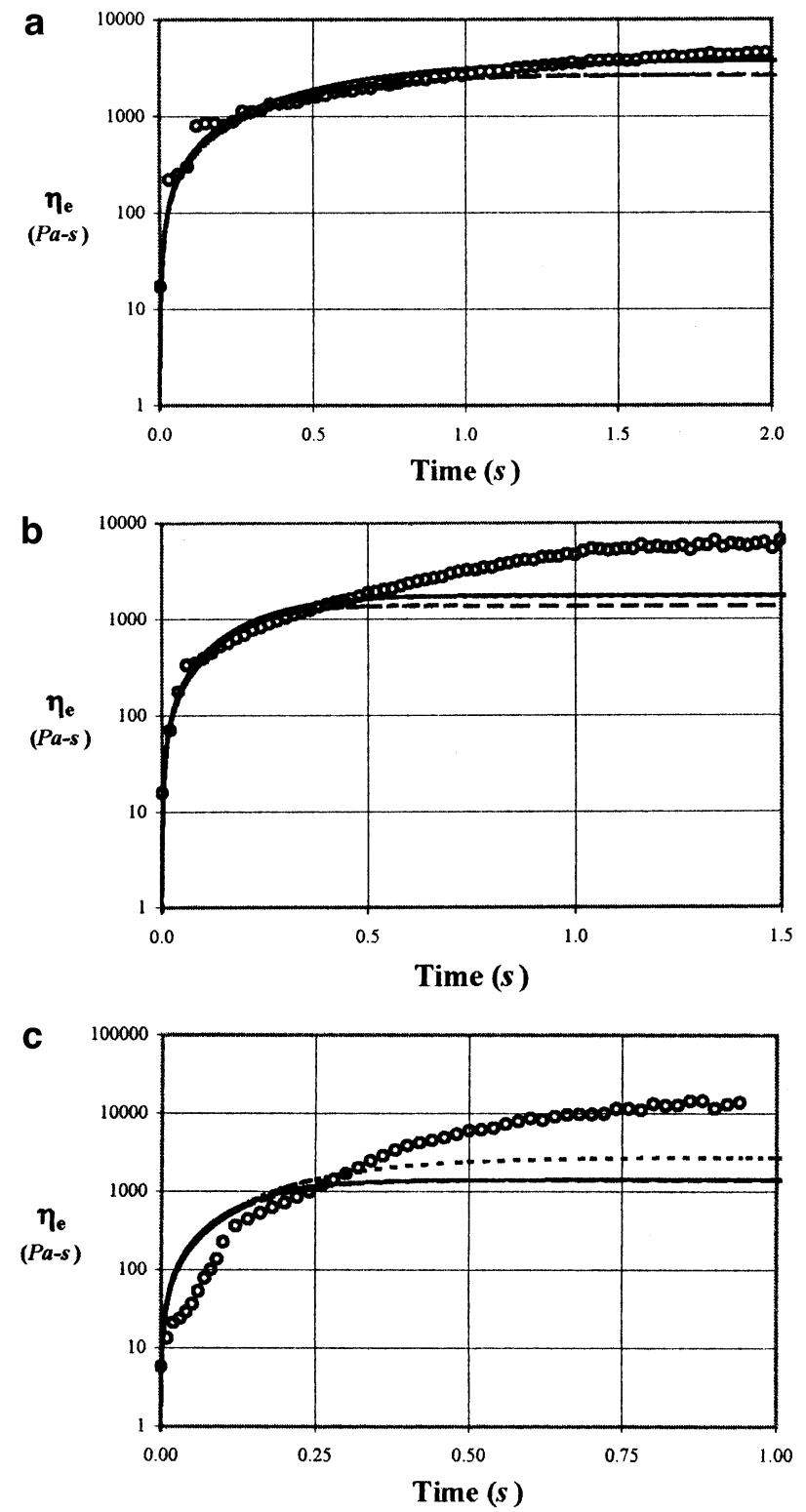

Figure 13. "Extensional viscosity" vs time in startup fl ow for the $3.9 \mathrm{M} 10.0 \mathrm{wt} \%$ solution for (a) $\dot{\epsilon}=1.12 \mathrm{~s}^{-1}$, (b) $\dot{\epsilon}=3.49$ $\mathrm{s}^{-1}$, and (c) $\dot{\epsilon}=7.19 \mathrm{~s}^{-1}:$ (O) experimental data, ( - ) basic DEMG model, $(--)$ MLD/Milner-McLeish CLF model, and (- - -) MLD/Doi-Kuzuu CLF model.

(or more accurately, the strain) associated with the transient stress growth. In this respect, the overall time to steady state predicted by all three versions of the model is too short, which is most likely a consequence of the fact that the estimated values of the Rouse and reptation time scales are too short, due to the fact that the nonlinear model does not include DCR. Referring back to the results in Table 2, the two sets of linear viscoelastic fits to our data using the LihktmanMcLeish model shows that there is approximately a factor of 2-3 increase in the estimates of these time scales when DCR is included in the linear viscoelastic model.

The deviation between the transient behavior pre dicted by the MLD model and the data thatoccurs at short times [evident particularly in Figure 13c] may be due partially to the fact that the startup process for the filament-stretching device does not lead instantaneously to a constant strain rate uniaxial extensional flow. 
Table 3. Solution Parameters for PS3 $\left(0.076 \mathrm{~g} / \mathrm{cm}^{3}\right.$ of $2.89 \mathrm{M}$ Polystyrene in a Mixed Solvent of Toluene and Styrene Oligomer)

\begin{tabular}{|c|c|c|c|c|c|c|c|c|c|c|}
\hline $\begin{array}{l}\text { estimation } \\
\text { method }\end{array}$ & $\left(\mathrm{Me}_{\mathrm{e}}\right)_{\mathrm{sol}}$ & $\begin{array}{l}\mathrm{G}_{\mathrm{N}}^{0} \\
(\mathrm{~Pa})\end{array}$ & Z & $\begin{array}{l}\left(\tau_{\mathrm{R}}\right)_{\mathrm{DEMG}} \\
(\mathrm{s})\end{array}$ & $\begin{array}{c}\left(\tau_{\mathrm{d}, 0}\right)_{\text {DEMG }} \\
(\mathrm{s})\end{array}$ & $\begin{array}{l}\left(\tau_{\mathrm{R}}\right)_{\mathrm{MM}} \\
(\mathrm{s})\end{array}$ & $\begin{array}{l}\left(\tau_{\mathrm{d}, 0}\right)_{\mathrm{MM}} \\
(\mathrm{s})\end{array}$ & $\begin{array}{l}\left(\tau_{\mathrm{R}}\right)_{\mathrm{DK}} \\
(\mathrm{s})\end{array}$ & $\begin{array}{l}\left(\tau_{\mathrm{d}, 0}\right)_{\mathrm{DK}} \\
(\mathrm{s})\end{array}$ & $\begin{array}{c}\text { stress-optical } \\
\text { coeff }(\mathrm{Pa})\end{array}$ \\
\hline $\begin{array}{l}\text { Yavich et al.a } \\
\text { current paper }\end{array}$ & $\begin{array}{l}269267 \\
210949\end{array}$ & $\begin{array}{l}708.8 \\
711\end{array}$ & $\begin{array}{l}10.0 \\
13.7\end{array}$ & $\begin{array}{l}0.456 \\
0.108\end{array}$ & $\begin{array}{c}13.7 \\
4.44\end{array}$ & 0.302 & 12.4 & 0.537 & 22.1 & $\begin{array}{l}-5.0 \times 10^{-9} \\
-3.38 \times 10^{-9}\end{array}$ \\
\hline
\end{tabular}

a Original paper.

\section{Comparisons with Previous Studies}

We note that the experimental results obtained here are similar in some respects to the birefringence data obtained earlier by Yavich et al. ${ }^{39}$ for steady 2D mixed type strong flows in a two-roll mill. In the original study, these data were compared to predictions using the DEMG model without either CLF or CCR and with model parameters obtained by more "ad hoc" and less reliable procedures than those discussed in section 4. While the results were qualitatively reasonable, it is of interest to see whether the nature of the fit is changed when more advanced models and better methods for parameter estimation are brought to bear. This is not simply a matter of adding another data set, as there are several fundamental differences between the uniaxial extensional flow studied in the previous sections and the more complicated 2D flows that are generated in the two-roll mill. The most important of these is that the two-roll mill flows are "mixed-type" strong flows rather than purely extensional flows. The designation as a "strong" flow means that material points separate exponentially in time, just as they do for a purely extensional flow (in contrast to a simple shear or viscometric flow). On the other hand, they are "mixedtype" in the sense that they contain both pure straining (or extensional) and vorticity components. Thus, an additional factor is that the orientation of the polymer chain relative to axes fixed in the flow is not known a priori. Consequently, successful predictions require not only accurate predictions of chain stretch but also chain orientation, which cannot be established in a purely extensional flow.

In this section, we revisit the data from Yavich et al. ${ }^{39}$ Our objective is to provide a test of the MLD model for this class of flows with model parameters recalculated following the procedures of section 4 . We do not discuss the details of the experiments, as these are available in the published paper of Yavich et al. Here, we note only that the polymer used was a $0.076 \mathrm{~g} / \mathrm{cm}^{3}$ solution of $2.89 \times 10^{6}$ molecular weight polystyrene in a toluene/ oligomer solvent (we denote this solution as PS3). The measurements were made at the central stagnation point of the two-roll mill, where the flow is $2 \mathrm{D}$ with a ratio of strain rate to vorticity (which can be varied) specified by a parameter $\lambda$, according to

$$
\frac{\|\mathbf{E}\|}{\|\Omega\|} \equiv \frac{1+\lambda}{1-\lambda}
$$

Data were obtained for three different configurations of the two-roll mill, corresponding to $\lambda=0.0196,0.06$, and 0.15 for Newtonian fluids. Because the polymer solution is viscoelastic, however, both the flow type and the magnitude of the velocity gradient are different than they would be for a Newtonian fluid. Thus, to make predictions via the MLD or any related model, one must actually measure these flow parameters and then compare the predicted and measured stress (or birefringence in this case) using the measured flow parameters as
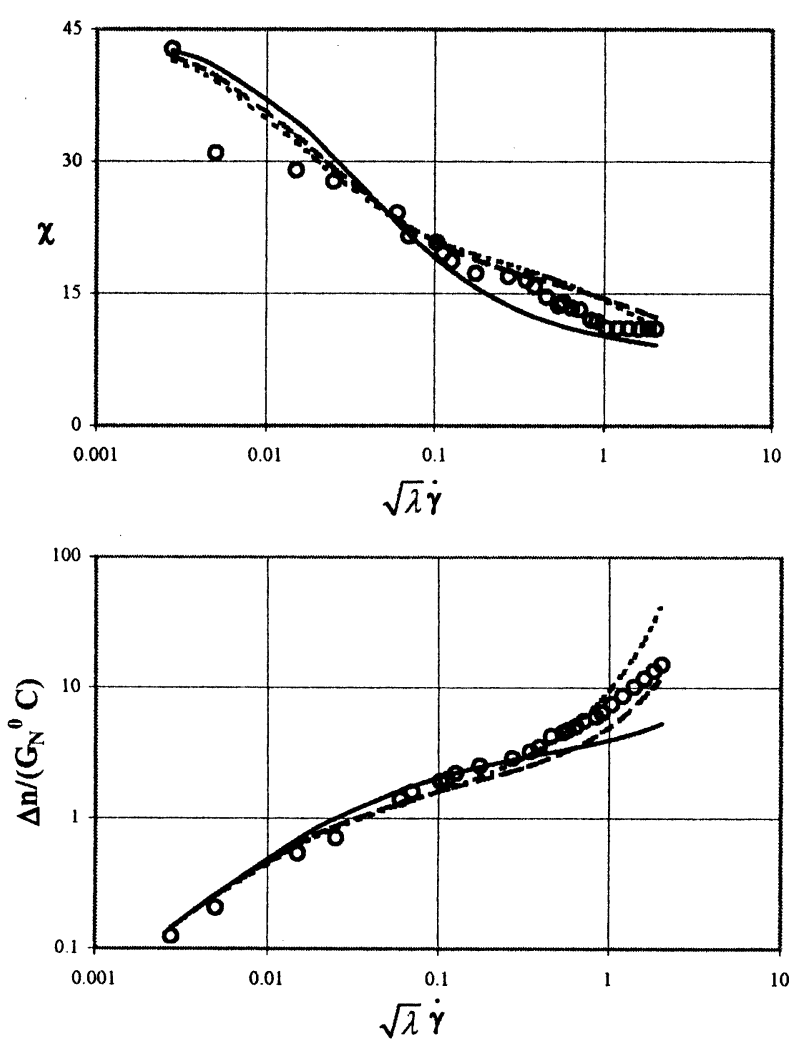

Figure 14. (a) Orientation angle and (b) dimensionless birefringence vs the principal eigenvalue of the velocity gradient tensor in a mixed-type flow generated in a two-roll mill with a flow type parameter $\lambda=0.0196$. The figure shows (O) experimental data, $(-)$ basic DEMG model, $(--)$ MLD/ Milner-McLeish CLF model, and (- - ) MLD/Doi-Kuzuu CLF model. The fluid is a $2.89 \mathrm{M}$ polystyrene solution with properties shown in Table 3.

inputs to the model. The necessary flow data was obtained by Yavich et al. using a dynamic light scattering technique. ${ }^{70}$

Linear viscoelastic data from the original paper of Yavich et al. ${ }^{39}$ was used to determine model parameters following the procedures described in section 4 (assuming that the toluene/oligomer mixture is a good solvent). The results are listed in Table 3, together with the parameter values that were originally estimated by Yavich et al. In the original study, the plateau modulus and the number of entanglements per chain were obtained from the scaling correlations of Osaki et al. ${ }^{61}$ for polystyrene in a good solvent, while the disengagement ti me for reptation, $\tau_{\mathrm{d}}$, was estimated directly from the crossing point for $\mathrm{G}^{\prime}$ and $\mathrm{G}^{\prime \prime}$ in the linear viscoelastic regime without any correction for the effect of CLF or DCR. The Rouse time, $\tau_{R}$, was then cal culated as $\tau_{d} / 3 Z$. Comparing the two sets of parameters, we see that the estimates of the plateau modulus are virtually identical; however, the Osaki correlation relating $\mathrm{Me}_{\mathrm{e}}$ and $\mathrm{G}_{\mathrm{N}}^{0}$ neglects the $4 / 5$ factor discussed earlier, causing the estimate of $Z$ in Yavich et al. to be smaller than the current estimate. We also see that the Rouse and 

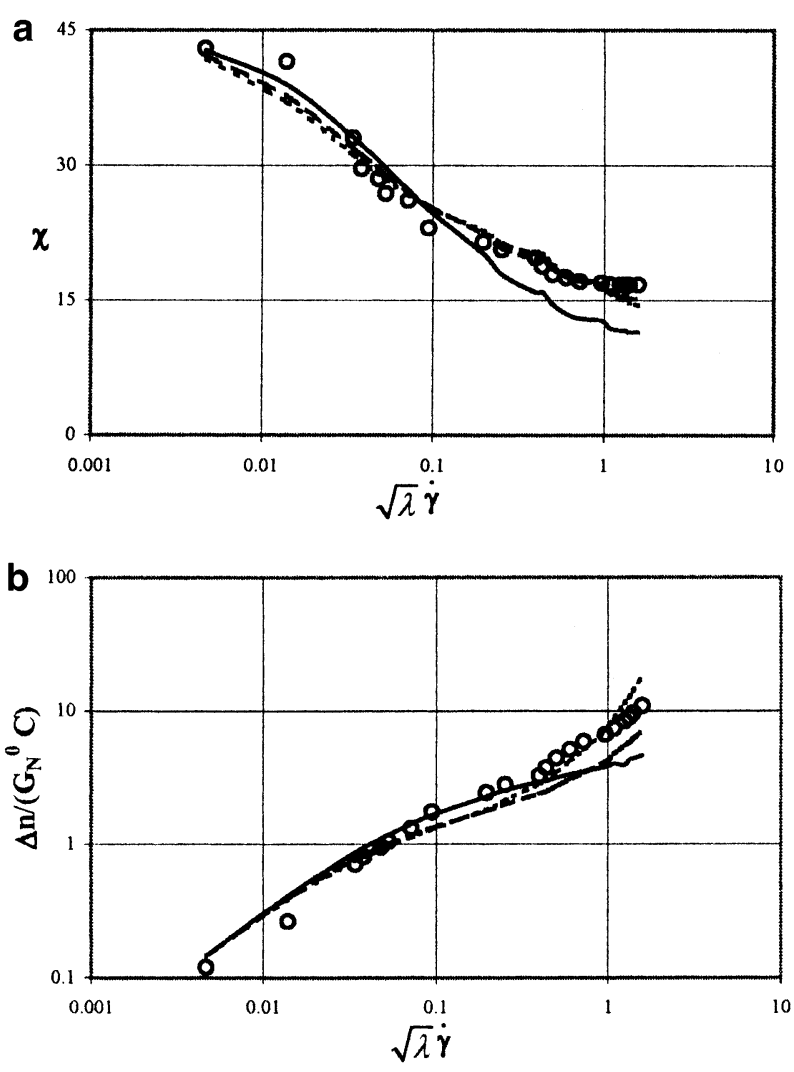

Figure 15. (a) Orientation angle and (b) dimensionless birefringence vs the principal eigenvalue of the velocity gradient tensor in a mixed-type flow generated in a two-roll mill with a flow type parameter $\lambda=0.06$. The figure shows (O) experimental data, (-) basic DEMG model, $(--)$ MLD/ Milner-McLeish CLF model, and (- - ) MLD/Doi-Kuzuu CLF model. The fluid is a $2.89 \mathrm{M}$ polystyrene solution with properties shown in Table 3.

reptation times used by Yavich et al. for DE MG calculations are similar to the current estimates including CLF (taking into account the change in Z).

With a new set of model parameters available, predictions for the birefringence and the average polymer orientation were carried out for the basic DEMG model, and for the full MLD model using both Milner-McLeish and Doi-Kuzuu versions of CLF with the parameters indicated in Table 3. Results are shown in Figures 1416 for the three configurations of the two roll mill reported in the original study. We reiterate that the model calculations are based on the measured flow data. Although the predicted results are plotted as smooth continuous curves, the velocity gradient data is only available at the same discrete set of $\dot{\gamma}$ values as the experimental points. The curves simply connect the computed values without smoothing, which explains the apparent bumps seen in some of the plots. Although it is not directly rel evant to the plots, we should al so point out that the principal eigenvalue of the velocity gradient tensor in 2D mixed-type flows becomes $\dot{\gamma} \sqrt{\lambda}$, and thus the magnitude of the vel ocity gradient where one should expect to see the onset of chain stretching is increased relative to the critical strain rate in a purely extensional flow by the factor $1 / \sqrt{\lambda}$.

To compare the measured and predicted birefringence shown in Figures 14-16, the experimental data must be scaled by the product of the plateau modulus $\mathrm{G}_{\mathrm{N}}^{0}$ and the stress-optical coefficient C. ${ }^{12}$ In the present a
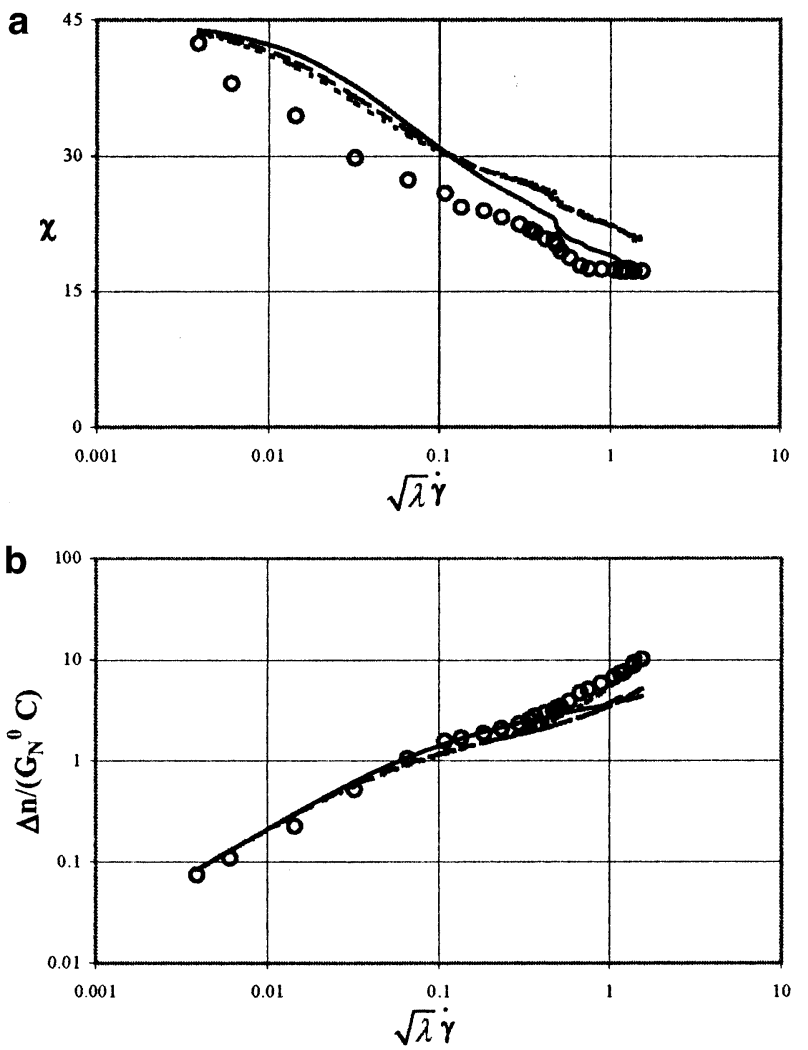

Figure 16. (a) Orientation angle and (b) dimensionless birefringence vs the principal eigenvalue of the velocity gradient tensor in a mixed-type flow generated in a two-roll mill with a flow type parameter $\lambda=0.15$. The figure shows (O) experimental data, $(-)$ basic DEMG model, $(--)$ MLD/ Milner-McLeish CLF model, and (- - -) MLD/Doi-Kuzuu CLF model. The fluid is a $2.89 \mathrm{M}$ polystyrene solution with properties shown in Table 3.

work, we use a value of $\mathrm{C}=-3.38 \times 10^{-9} \mathrm{~Pa}$. That is based on direct measurements of the stress and birefringence for polystyrene in a good solvent. ${ }^{38}$ As shown in Table 3, Yavich et al. used a somewhat larger value taken from the literature.

Let us first consider results for the smallest flow type parameter, $\lambda=0.0196$, shown in Figure 14. Looking initially at the orientation angle, we see that the inclusion of CCR and CLF in the form proposed by Mead et al. seems to improve the orientation angle predictions over the results for the basic DE MG model. In fact, other than one point at the next to smallest value of $\sqrt{\lambda} \dot{\gamma}$, the MLD predictions with the current parameter values are very good. Since the measurement of the orientation angle via birefringence is susceptible to low signal strength at low $\dot{\gamma}$, it is more likely the data point is at fault rather than the prediction. Similarly, the MLD prediction of the birefringence with current parameter values is virtually perfect up to the largest values of $\sqrt{\lambda} \dot{\gamma}$, where there is a slight deviation. Furthermore, referring back to the original paper, we see that that there is also a modest though significant improvement in the predictions based on the current rather than the original Yavich parameter values.

The results for $\lambda=0.06$, shown in Figure 15 , are qualitatively similar, with the MLD model using current parameter values producing the most accurate predictions. The MLD predictions of the orientation angleand the bir efringence predictions are again amazingly good when compared with the data scaled with the current 
estimate of the stress-optical coefficient, especially with the Doi-Kuzuu version of CLF .

Finally, we consider the results for $\lambda=0.15$, shown in Figure 16. The bir efringence comparisons in this case are again basically perfect for the MLD/Doi-Kuzuu CLF model with current parameter values. However, the comparisons between measured and predicted values of the orientation angle are less satisfactory. Both the DEMG and MLD models seem to predict too little rotation away from the principal strain rate direction $\left(45^{\circ}\right)$. We do not understand the origin of this discrepancy, and thus we are uncertain of its significance. Superficially, one would tend to think that the prediction of orientation angle would improve as $\lambda$ increases toward the limit $\lambda=1$, which corresponds to a purely extensional planar flow for which the orientation angle is fixed at $45^{\circ}$ (like the case of the uniaxial extension considered in the main body of this paper). Experimental measurements also become easier as $\lambda$ increases, because the gap between the cylinders in the two-roll mill increases relative to their radius, facilitating optical access. Furthermore, the fact that the birefringence is so accurately reproduced seems to suggest that the flow characterization obtained from the light scattering must be quite good. Consequently, we cannot explain the failure of the MLD model to accurately predict the orientation angle for $\lambda=0.15$.

Despite this one issue, however, we conclude that the MLD model, especially with Doi-Kuzuu CLF, generally provides very satisfactory predictions for both the orientation angle and the bi refringence for these mixedtype flows. Indeed, the predictions are better than we should expect given the absence of mechanisms such as DCR.

\section{Conclusions}

To the best of our knowledge, we have obtained the first reliable data on the transient evolution of the extensional stresses and the steady state uniaxial extensional viscosity for highly entangled, monodisperse polymer solutions in a uniaxial extensional flow. In doing so, we have provided for the first time a unique basis with which to test the models that have recently been proposed to describe the nonlinear dynamical behavior of entangled polymers based on extensions of the Doi-Edwards reptation model to account for chain stretching, contour length fluctuations, and convective constraint release.

The results are in rather poor agreement with the model of M ead, Larson, and Doi, ${ }^{1}$ at least for this class of flows in the range of strain rates that we have been able to achieve in the filament stretching device. Specifically, the steady state predictions for the extensional viscosity are lower than the data in all cases and are lower by large amounts depending on the strain rate that is chosen. We surmise that the discrepancies may be mainly due to the fact that the MLD model does not include DCR and the corresponding dual constraint release version of the CCR mechanism. The inclusion of these mechanisms will have two effects: the model predictions will be changed directly, but more importantly, the estimates of time scales will be changed by inclusion of the DCR mechanism.

In addition, we have applied the same methodology to steady 2D mixed-type strong flows, where experimental birefringence data is available from an earlier investigation. ${ }^{39} \mathrm{Here}$, an additional factor is the orienta- tion of the polymer chain relative to axes fixed in the flow. For a variety of flow types, the birefringence is predicted very accurately. Similarly, the orientation angle is accurately calculated for the two flows nearest to simple shear flow, where the model has previously been tested, ${ }^{1}$ but less well predicted for the flow that is furthest from simple shear. Until more experimental data is available, it is unclear whether this discrepancy is due to a limitation of the model or inaccuracy in the data.

It seems surprising that the MLD model appears to provide better agreement with the data for the mixedtype flows, where one must accurately predict both the orientation angle and the bi refringence (or stress), than for the apparently simpler case of uniaxial extensional flow, where the orientation angle is known. The only point that we can make is that the model parameters were fit to linear viscoelastic data in simple shear flow, and at least for the comparisons that we have made in this paper, the most accurate predictions correspond to the flows that are most similar to shear. While it is probably premature to draw any conclusion from this observation until we have compared the data to a model incorporating all of the currently proposed mechanisms (DCR and dual convective constraint release in particular), it is nevertheless a provocative result.

Acknowledgment. J .P.O. and L.G.L. wish to acknowledge the MRL Central Facilities at UCSB, supported by the National Science Foundation under Award No. DMR96-32716, which provided computing resources for the transient uniaxial extensional flow calculations. The participation of J .P.O. and L.G.L. in this project has also been supported by the Solid State Chemistry and Polymers Program of the NSF under Award No. DMR00-89124. J .P.O. would also like to acknowledge the computing resources at the University of Virginia used for steady state model calculations. The rheology research group at Monash University acknowledges a grant from the Australian Research Council. The authors also acknowledge useful discussions with Professors R. G. Larson, T. C. B. McLeish, and S. T. Milner as well as Dr. Alexei Lihktman. The final draft of this paper was written while T.S. and L.G.L. were participating in the complex fluids program of the ITP at UCSB.

\section{References and Notes}

(1) Mead, D. W.; Larson, R. G.; Doi, M. Macromolecules 1998, 31, 7895-7914.

(2) Doi, M.; Edwards, S. F. The Theory of Polymer Dynamics; Oxford University Press: Oxford, England, 1986.

(3) Doi, M.; Edwards, S. F. J . Chem. Soc., Faraday Trans. 2 1978, 74, 1789-1801.

(4) Doi, M.; Edwards, S. F. J . Chem. Soc., Faraday Trans. 2 1978, 74, 1802-1817.

(5) Doi, M.; Edwards, S. F. J . Chem. Soc., Faraday Trans. 2 1978, 74, $1818-1832$.

(6) Doi, M.; Edwards, S. F. J . Chem. Soc., Faraday Trans. 21979 $75,38-54$.

(7) Leal, L. G.; Oberhauser, J . P. Kor.-Austr. J . Rheol . 2000, 12, $1-25$.

(8) Watanabe, H. Prog. Polym. Sci. 1999, 24, 1253-1403.

(9) McLeish, T. C. B. Adv. Phys. 2002, 51, 1379-1527.

(10) Marrucci, G.; Grizzuti, N. Gazz. Chim. Ital. 1988, 118, 179185.

(11) Pearson, D.; Herbolzheimer, E.; Grizzuti, N.; Marrucci, G. J . Polym. Sci., Part B: Polym. Phys. 1991, 29, 1589-1597.

(12) Mead, D. W.; Leal, L. G. Rheol. Acta 1995, 34, 339-359.

(13) Mead, D. W.; Yavich, D.; Leal, L. G. Rheol. Acta 1995, 34, $360-383$. 
(14) Doi, M. J . Polym. Sci., Polym. Phys. Ed. 1983, 21, 667-684.

(15) Milner, S. T.; McLeish, T. C. B. Phys. Rev. Lett. 1998, 81, $725-728$.

(16) Milner, S. T. J . Rheol. 1996, 40, 303-315.

(17) Rubinstein, M.; Colby, R. H.J . Chem. Phys. 1988, 89, 52915306.

(18) Viovy, J . L.; Rubinstein, M.; Colby, R. H. Macromolecules 1991, 24, 3587-3596.

(19) Ianniruberto, G.; Marrucci, G. J . Non-Newtonian Fluid Mech. 1996, 65, 241-246.

(20) I anniruberto, G.; Marrucci, G. Proc. Int. Congr. Rheol. 2000, $2,102-104$.

(21) Marrucci, G. J . Non-Newtonian Fluid Mech. 1996, 62, 279289.

(22) Likhtman, A. E.; Milner, S. T.; McLeish, T. C. B. Phys. Rev. Lett. 2000, 85, 4550-4553.

(23) Milner, S. T.; McLeish, T. C. B.; Likhtman, A. E. J . Rheol. 2001, 45, 539-563.

(24) Marrucci, G.; I Ianniruberto, G. J . Non-Newtonian Fluid Mech. 1999, 82, 275-286.

(25) Mhetar, V.; Archer, L. A. J . Non-Newtonian Fluid Mech. 1999, 81, 71-81.

(26) Archer, L. A. J . Rheol. 1999, 43, 1555-1571.

(27) Wagner, M. H.; Schaeffer, J . Rheol . Acta 1994, 33, 506-516.

(28) Wagner, M. H.J . Non-Newtonian Fluid Mech. 1997, 68, 169171.

(29) Osaki, K.; Kimura, S.; Kurata, M. J . Polym. Sci., Polym. Phys. Ed. 1981, 19, 517-527.

(30) Archer, L. A.; Chen, Y. L.; Larson, R. G. J . Rheol. 1995, 39, $519-525$

(31) Osaki, K.; Inoue, T.; Uematsu, T. J . Polym. Sci. Part B: Polym. Phys. 2000, 38, 3271-3276.

(32) Osaki, K.; I noue, T.; I somura, T.J . Polym. Sci. Part B: Polym. Phys. 2000, 38, 1917-1925.

(33) Menezes, E. V.; Graessley, W. W. J . Polym. Sci., Polym. Phys. Ed. 1982, 20, 1817-1833.

(34) Pearson, D. S.; Kiss, A. D.; Fetters, L. J .; Doi, M. J . Rheol 1989, 33, 517-535.

(35) Zebrowski, B. E.; Fuller, G. G. J . Polym. Sci., Polym. Phys. Ed. 1985, 23, 575-589.

(36) Oberhauser, J. P.; Leal, L. G.; Mead, D. W. J . Polym. Sci. Part B: Polym. Phys. 1998, 36, 265-280.

(37) Oberhauser, J. P.; Pham, K. Q.; Leal, L. G. Manuscript in preparation-preprint available, 2002

(38) Oberhauser, J . Ph.D. Thesis, University of California at Santa Barbara, Santa Barbara, CA, 2001.

(39) Yavich, D.; Mead, D. W.; Oberhauser, J . P.; Leal, L. G. J Rheol. 1998, 42, 671-695.

(40) Sridhar, T.; Tirtaatmadja, V.; Nguyen, D. A.; Gupta, R. K.J . Non-Newtonian Fluid Mech. 1991, 40, 271-280.

(41) Tirtaatmadja, V.; Sridhar, T. J . Rheol. (U.S.A.) 1993, 37, $1081-1102$.

(42) Brandrup, J .; Immergut, E. H.; Grulke, E. A.; Eds. Polymer Handbook, 4th ed; J ohn Wiley and Sons: New York, 1999.
(43) Orr, N. V.; Sridhar, T. J . Non-Newtonian Fluid Mech. 1999, 82, 203-232.

(44) Gupta, R. K.; Nguyen, D. A.; Sridhar, T. Phys. Fluids 2000, $12,1296-1318$

(45) Sridhar, T. Kor.-Austr. J . Rheol. 2000, 12, 39-53.

(46) McKinley, G. H.; Sridhar, T. In Annual Review of Fluid Mechanics; Annual Reviews Press: Palo Alto, CA, 2002; Vol. 34, pp 375-415.

(47) Doi, M.; Kuzuu, N. Y. J . Polym. Sci., Polym. Lett. Ed. 1980, $18,775-780$.

(48) Marrucci, G.; Greco, F.; I anniruberto, G. J . Rheol. 2000, 44, 845-854.

(49) Likhtman, A. E.; McLeish, T. C. B. Macromol ecules 2002, 35 , $6332-6343$

(50) Doi, M.; Graessley, W. W.; Helfand, E.; Pearson, D. S. Macromolecules 1987, 20.

(51) des Cloizeaux, J . Europhys. Lett. 1988, 5, 437-442.

(52) des Cloizeaux, J. Macromol ecules 1990, 23, 3992-4006.

(53) des Cloizeaux, J. Macromolecules 1990, 23, 4678-4687.

(54) Tsenoglou, C. Macromolecules 1991, 24, 1762-1767.

(55) Tsenoglou, C. J . Polym. Sci., Part B: Polym. Phys. 1988, 26 2329-2339.

(56) Tsenoglou, C. Polym. Prepr. 1987, 28, 185-188.

(57) Fetters, L. J .; Lohse, D. J .; Richter, D.; Witten, T. A.; Zirkel, A. Macromolecules 1994, 27, 4639-4647.

(58) Graessley, W. W. J . Polym. Sci., Polym. Phys. Ed. 1980, 18 27-34.

(59) Ferry, J . D. Viscoelastic properties of polymers, 3d ed; Wiley: New York, 1980.

(60) de Gennes, P.-G. Scaling Concepts in Polymer Physics; Cornell University Press: Ithaca, NY, and London, 1979.

(61) Osaki, K.; Nishimura, Y.; Kurata, M. Macromolecules 1985 $18,1153-1157$.

(62) Raju, V. R.; Menezes, E. V.; Graessley, W. W.; Fetters, F. J Macromolecules 1981, 14, 1668-1672.

(63) Osaki, K.; Inoue, T.; U ematsu, T.; Yamashita, Y. J . Polym. Sci., Part B: Polym. Phys. 2001, 39, 1704-1712.

(64) Harrison, G. M. Ph.D. Thesis, University of California, Santa Barbara, Santa Barbara, CA, 1997; p 284.

(65) Harrison, G. M.; Leal, L. G. 2002, Manuscript in preparation.

(66) I anniruberto, G.; Marrucci, G. J . Non-Newtonian Fluid Mech. 2000, 95, 363-374.

(67) Pattamaprom, C.; Larson, R. G.; Van Dyke, T. J . Rheol . Acta 2000, 39, 517-531.

(68) Pattamaprom, C.; Larson, R. G. Macromolecules 2001, 34, $5229-5237$

(69) Pattamaprom, C.; Larson, R. G. Rheol. Acta 2001, 40, 516532.

(70) Wang, J J J .; Yavich, D.; Leal, L. G. Phys. Fluids 1994, 6, 3519-3534.

MA0118623 